\title{
Spectrophotometry of galaxies in the Virgo cluster ${ }^{\star} \star \star$
}

\author{
II. The data \\ G. Gavazzi ${ }^{1}$, A. Zaccardo ${ }^{1}$, G. Sanvito ${ }^{1}$, A. Boselli ${ }^{2}$, and C. Bonfanti ${ }^{1}$ \\ 1 Università degli Studi di Milano - Bicocca, Piazza delle scienze 3, 20126 Milano, Italy \\ 2 Laboratoire d'Astrophysique de Marseille, Traverse du Siphon, 13276 Marseille Cedex 12, France
}

Received 23 July 2003 / Accepted 16 December 2003

\begin{abstract}
Drift-scan mode (3600-6800 A) spectra with $500<R<1000$ resolution are presented for 333 galaxies members of nearby clusters, covering the whole Hubble sequence. The majority (225) were obtained for galaxies in the Virgo cluster where a completeness of $36 \%$, if all Hubble types are considered, and of $51 \%$, restricting to late-types, was reached at $m_{p} \leq 16$. Our data can be therefore considered representative of the integrated spectral properties of giant and dwarf galaxies in this cluster. Intensities and equivalent widths $(E W s)$ are derived for the principal lines, both in emission and in absorption. Deblending of the underlying absorption from emission was achieved in most cases.
\end{abstract}

Key words. galaxies: clusters: individual: Virgo - techniques: spectroscopic - catalogs

\section{Introduction}

Local galaxies are the relics of evolutionary processes that took place in the universe from the early collapse of primordial matter fluctuations up to the present cosmological epoch. Such processes have not yet been convincingly unveiled, in spite of an increasing observational effort involving today's major observational facilities. A satisfactory characterization of the properties of local galaxies is building up only recently, as data obtained through a variety of observational windows of the electromagnetic spectrum are being gathered (see Kennicutt 1998; Roberts \& Haynes 1994; Gavazzi et al. 1996; Tuffs et al. 2002; Popescu et al. 2002). Complete imaging data sets taken in a broad frequency range are becoming available owing to extensive observational campaigns, e.g. 2MASS (Jarrett et al. 2003), SLOAN (Stoughton et al. 2002), SINGS (Kennicutt et al. 2003) just to mention few.

As far as nearby clusters, such as the Virgo cluster and the Coma supercluster, multifrequency data for over 3000 galaxies

Send offprint requests to: G. Gavazzi,

e-mail: giuseppe.gavazzi@mib.infn.it

* Based on observations collected at the Observatoire de Haute Provence (OHP) (France), operated by the CNRS, at the European Southern Observatory (proposals 66.B-0026; 68.B-0505), at the Loiano telescope belonging to the University of Bologna (Italy) and at the Observatorio Astronomico National de San Pedro Martir (Mexico).

$\star \star$ Full Tables 6-8 and full Fig. 19 (in FITS) are only available in electronic form at the CDS via anonymous ftp to cdsarc.u-strasbg.fr $(130.79 .128 .5)$ or via

http://cdsweb.u-strasbg.fr/cgi-bin/qcat?J/A+A/417/499 are collected and distributed via the WEB site "GOLDMine" (Gavazzi et al. 2003).

Spectroscopic data are equally invaluable sources of information, but are more difficult to obtain and more time consuming. The spectroscopic characterization of the stellar continua provide us with "clocks" on stellar populations, while line indices enable us to quantify the chemical evolution of the stars and of the interstellar medium in galaxies. However much less extensive surveying was carried out in the spectroscopic than in the imaging mode, if one excludes the pioneering work of Kennicutt (1992, K92 hereafter) who first tried to assess the systematic spectral properties of nearby galaxies along the Hubble sequence and of Jansen et al. (2000) who extended the spectral survey of $\mathrm{K} 92$ to a large sample of isolated galaxies, later analyzed by Stasinska \& Sodre (2001). These spectroscopic surveys were carried out in the drift-scan mode, i.e. with the slit sliding over the whole galaxy area. Spectra taken in this way are representative of the mean galaxies, unlike most long slit observations which are dominated by the nuclear light.

In 1998, inspired by the work of K92 and of Jansen et al. (2000) we initiated a long term project aimed at characterizing spectroscopically the galaxies in the nearest rich cluster: the Virgo cluster. In Paper I of this series (Gavazzi et al. 2002a) we analyzed the spectral continua based on preliminary 124 spectra obtained until 2001. Here we present the full set of 333 spectra obtained so far (spring 2003). They are available in JPG and FITS format at the WEB site GOLDMine (http://goldmine.mib.infn.it) (Gavazzi et al. 2003). Their analysis is postponed to Paper III (in preparation).

The present paper is organized as follows: Sect. 2 describes the surveyed sample, the observational and data reduction 
Table 1. The spectrograph characteristics.

\begin{tabular}{lccccccccc}
\hline \hline Telescope & Run & Spectrograph & $\begin{array}{c}\text { Disp } \\
\AA / \mathrm{mm}\end{array}$ & $\begin{array}{c}\text { Disp } \\
\AA / \mathrm{pix}\end{array}$ & $\begin{array}{c}\Delta \lambda \\
\AA\end{array}$ & CCD & $\begin{array}{c}\text { Pix } \\
\mu \mathrm{m}\end{array}$ & $\begin{array}{c}\text { Spat. scale } \\
\text { "/pix }\end{array}$ & $\begin{array}{c}\text { Slit } \\
\text { arcsec }\end{array}$ \\
\hline OHP/1.93 & 1998 & CARELEC & 260 & 7.0 & $3600-7200$ & $512 \times 512$ TEK & 27 & 1.17 & $300 \times 2.5$ \\
OHP/1.93 & 1999 & CARELEC & 133 & 1.8 & $3400-7000$ & $2048 \times 1024$ EEV & 13.5 & 0.58 & $300 \times 2.5$ \\
OHP/1.93 & 2000 & CARELEC & 133 & 1.8 & $3400-7000$ & $2048 \times 1024$ EEV & 13.5 & 0.58 & $300 \times 2.5$ \\
OHP/1.93 & 2001 & CARELEC & 133 & 1.8 & $3400-7000$ & $2048 \times 1024$ EEV & 13.5 & 0.58 & $300 \times 2.5$ \\
OHP/1.93 & 2002 & CARELEC & 133 & 1.8 & $3400-7000$ & $2048 \times 1024$ EEV & 13.5 & 0.58 & $300 \times 2.5$ \\
OHP/1.93 & 2003 & CARELEC & 133 & 1.8 & $3400-7000$ & $2048 \times 1024$ EEV & 13.5 & 0.58 & $300 \times 2.5$ \\
ESO/3.6(LD) & 2001 & EFOSC2 & 135 & 4.0 & $3380-7520$ & $2048 \times 2048$ LOR & 15 & 0.16 & $300 \times 1.5$ \\
ESO/3.6(HD) & 2001 & EFOSC2 & 67 & 2.0 & $4700-6770$ & $2048 \times 2048$ LOR & 15 & 0.16 & $300 \times 1.5$ \\
ESO/3.6(LD) & 2002 & EFOSC2 & 135 & 4.0 & $3380-7520$ & $2048 \times 2048$ LOR & 15 & 0.16 & $300 \times 1.5$ \\
SPM/2.1 & 2002 & Boller\&Chivens & 125 & 3.0 & $3900-7000$ & $1024 \times 1024$ SITE3 & 24 & 0.96 & $300 \times 2.0$ \\
LOI/1.52 & 2003 & BFOSC & 198 & 4.0 & $3600-8900$ & $1300 \times 1340$ EEV & 20 & 0.58 & $300 \times 2.0$ \\
\hline
\end{tabular}

techniques. Section 3 gives the details of the line measurements, including the deblending of unresolved lines and the separation of emission from underlying absorption lines. The derived spectral parameters are given in Sect. 4 and briefly summarized in Sect. 5.

\section{Observations}

Long-slit spectra of 333 galaxies, obtained during approximately 50 nights distributed in 6 years (1998-2003) using the $1.93 \mathrm{~m}$ telescope of the Observatoire de Haute Provence (OHP), the ESO/3.6 m telescope, the Loiano/1.52 m telescope and the San Pedro Martir (SPM) $2.1 \mathrm{~m}$ telescope are presented. The observations were taken in the "drift-scan" mode: i.e. with the slit, generally parallel to the galaxy major axis, drifting over the optical surface of the galaxy ${ }^{1}$.

At ESO we set the guide velocity of the telescope such that during the integration time the slit slides one time through the full length of the galaxy. At the remaining observatories the drifting was obtained by slewing manually several times the telescope between two extreme positions checked on one offset star or on the galaxy itself. Not unexpectedly spectra obtained in this way have lower $\mathrm{S} / \mathrm{N}$ ratio than traditional long-slit spectra of similar integration time, because a large fraction of the time is spent on low surface brightness regions. Our spectra cover the wavelength range $3600-6800 \AA$ (from [OII] to [SII]) with a resolution of $500<R<1000$. The spectrograph characteristics are given in Table 1. For 6 bright emission line galaxies observed at ESO we used both a low resolution grism and a high resolution red grism.

The observations at OHP, Loiano and San Pedro Martir were carried out in approximately $1.5-3$ arcsec seeing conditions, while subarcsecond conditions were often encountered at ESO. The present data, owing to the "drift-scan" method

\footnotetext{
${ }^{1}$ Galaxies with major axis $>5$ arcmin were observed with the slit perpendicular to the major axis. Few galaxies with both diameters larger than the slit length were observed. However most of the light from these objects comes from a region corresponding to half the (25th mag $\operatorname{arcsec}^{-2}$ ) diameters quoted in Table 2, thus well within the slit length.
}

are marginally affected by the seeing conditions. The OHP observations were sometimes taken through cirrus, otherwise in transparent or photometric conditions, while the observations obtained at ESO, SPM and Loiano were transparent or photometric. The spectrophotomectric standards Feige 34, Hz 44 and Hiltner 600 (ESO) were observed twice on each night.

\subsection{The sample}

Targets of the present spectrophotometric measurements were primarily selected from the Virgo Cluster Catalog (Binggeli et al. 1985: VCC). Among these we observed 225 objects $^{2}$. Limiting to the 621 galaxies with $m_{p} \leq 16$ which are Virgo cluster members $(V<3000$ or classified as possible members by Binggeli et al. 1985, 1993; Gavazzi et al. 1999), 223 have their spectra measured. At this limiting magnitude the completeness of our spectroscopic work is thus $36 \%$ and it increases to $46 \%$ at $m_{p} \leq 15$ and to $62 \%$ at $m_{p} \leq 14$, as listed in Table 3 . Most unobserved galaxies are $\mathrm{dE}$ and $\mathrm{E}$, therefore the completeness results significantly higher among late-type galaxies. In these morphological classes we covered more than $50 \%$ of the galaxies with $m_{p} \leq 16$. At the adopted distance of $17 \mathrm{Mpc}$ (or $\mu=31.1$ ) $m_{p}=16$ corresponds to $M_{p}=-15$, thus our survey can be considered as representative of the spectroscopic properties of late-type galaxies in the Virgo cluster including dwarf systems.

Observations of CGCG (Zwicky et al. 1961-68) galaxies with $m_{p} \leq 15.7$ in other nearby clusters (45 in Coma+A1367, 37 in Cancer, 8 in the A262 and Centaurus clusters and another 10 isolated objects) were taken as fillers when Virgo was not observable. These do not form a complete set.

Table 2 summarizes the number of obtained spectra in each run and cluster and the approximate seeing conditions.

\footnotetext{
2 Including 6 additional spectra taken with the William Herschel Telescope kindly provided to us by J. M. Vilchez (VCC 324, 334, $562,841,848$, and 2037) and 2 spectra taken from the spectral atlas of Kennicutt (1992) (VCC 355 e 1226) the total number of Virgo spectra is 233 .
} 
Table 2. The number of observed spectra.

\begin{tabular}{lccccccccc}
\hline \hline Telescope & Date & Virgo & Coma & A262 & Cancer & Centaurus & Other & Tot & Seeing $\left(^{\prime \prime}\right)$ \\
\hline OHP/1.93 & 5 Mar. 1998 & 9 & - & - & - & - & - & 9 & $2.0-3.0$ \\
OHP/1.93 & 9-15 Mar. 1999 & 22 & - & - & 2 & - & 2 & 26 & $2.0-3.0$ \\
OHP/1.93 & 1-6 Feb. 2000 & 27 & - & 8 & 16 & - & 2 & 53 & $2.0-3.0$ \\
OHP/1.93 & 19-25 Mar. 2001 & 24 & 5 & - & 1 & - & - & 30 & $2.0-3.0$ \\
OHP/1.93 & 7-13 Mar. 2002 & 51 & 4 & - & 4 & - & 1 & 60 & $2.0-3.0$ \\
OHP/1.93 & 25 Mar.-06 Apr. 2003 & 14 & 33 & - & - & - & - & 47 & $2.0-3.0$ \\
ESO/3.6(LD) & 23-25 Mar. 2001 & 41 & 1 & - & - & 8 & - & 50 & $0.5-2.0$ \\
ESO/3.6(HD) & 23-25 Mar. 2001 & $(6)$ & - & - & - & - & - & $(6)$ & $0.5-2.0$ \\
ESO/3.6(LD) & 15-16 Mar. 2002 & 33 & - & - & - & - & 5 & 38 & $0.5-1.0$ \\
SPM/2.1 & 17 Mar 2002 & 3 & 1 & - & - & - & - & 4 & $1.5-2.0$ \\
LOI/1.52 & Jan.-Feb. 2003 & 1 & 1 & - & 14 & - & - & 16 & $2.0-2.5$ \\
\hline Tot & & $225+(6)$ & 45 & 8 & 37 & 8 & 10 & $333+(6)$ \\
\hline
\end{tabular}

Table 3. Completeness of spectroscopic observations for Virgo cluster members and possible members regardless of their Hubble type and for late-type galaxies.

\begin{tabular}{lcccc}
\hline \hline$m_{p g}$ & $N$ VCC & With $z$ & $N$ Spectra & $\%$ \\
\hline$\leq 16$ all types & 621 & 568 & 223 & $(36)$ \\
$\leq 15$ all types & 430 & 427 & 198 & $(46)$ \\
$\leq 14$ all types & 252 & 252 & 157 & $(62)$ \\
$\leq 16$ late-type & 323 & 318 & 164 & $(51)$ \\
$\leq 15$ late-type & 244 & 244 & 149 & $(61)$ \\
$\leq 14$ late-type & 151 & 151 & 114 & $(75)$ \\
\hline
\end{tabular}

General parameters derived from the literature for galaxies in the observed sample, along with the log-book of the observations, are given in Table 6 , arranged as follows:

Column 1: galaxy designation.

Columns 2, 3: (J2000) celestial coordinates.

Column 4: heliocentric velocity (from this work or from the literature).

Column 5: cluster membership. The membership to the various sub-units within the Virgo cluster is according to Gavazzi et al. (1999).

Column 6: morphological type (from the VCC or from Gavazzi \& Boselli 1996).

Column 7: $\mathrm{S}=$ Seyfert, $\mathrm{L}=$ Liner H = HII (from NED).

Columns 8, 9: major and minor $B$ band optical diameters (in arcmin). These are consistent with the diameters given in the UGC.

Column 10: distance in Mpc. We assume a distance of $17 \mathrm{Mpc}$ for the members (and possible members) of Virgo cluster A, $22 \mathrm{Mpc}$ for Virgo cluster B, $32 \mathrm{Mpc}$ for objects in the $\mathrm{M}$ and $\mathrm{W}$ clouds. We adopt a distance of $65 \mathrm{Mpc}$ for A262 and of $33 \mathrm{Mpc}$ for the Centaurus cluster; 51-74 Mpc for the Cancer cluster, according to the membership to the individual sub-groups. Distances of 96 and 91.3 Mpc are assumed for Coma and A1367 respectively. We adopt $H_{o}=75 \mathrm{~km} \mathrm{~s}^{-1} \mathrm{Mpc}^{-1}$.

Columns 11-13: total apparent (uncorrected) $V, B$ and $H$ $(1.65 \mu \mathrm{m})$ magnitudes. These are magnitudes at the 25th mag $\operatorname{arcsec}^{-2}$ isophote obtained consistently with Gavazzi \& Boselli (1996).
Column 14: observing run.

Column 15: photometric quality: $\mathrm{P}=$ Photometric; $\mathrm{T}=$ transparent; $\mathrm{C}=$ thin Cirrus.

Column 16: dispersion.

Column 17: integration time (number of exposures $\times$ individual exposure time).

\subsection{Data reduction}

The reduction of the spectra was carried out using standard tasks in the IRAF package ${ }^{3}$. Visual inspection of the raw images provided us with a list of bad-pixel which were masked from the science frames. Bias subtraction and flat-field normalization was applied using median of several bias frames and exposures of quartz lamps. When at least three exposures were obtained for an object (see "number of exposures" in Col. 17 of Table 6), they were combined using a median filter, thus removing the cosmic rays. Otherwise the point-like ones were subtracted using COS MICRAY and the remaining extended features were removed under visual inspection of the spectra.

The $\lambda$ calibration was carried out using IDENTIFY REIDENTIFY - FITCOOR on exposures of He/Ar lamps and the calibration was transferred to the science frames using

\footnotetext{
${ }^{3}$ IRAF is the Image Analysis and Reduction Facility made available to the astronomical community by the National Optical Astronomy Observatories, which are operated by AURA, Inc., under contract with the US National Science Foundation. STSDAS is distributed by the Space Telescope Science Institute, which is operated by the Association of Universities for Research in Astronomy (AURA), Inc., under NASA contract NAS 5-26555.
} 
TRANS FORM. Typical errors on the dispersion solution are of few tenths of $\AA$, as confirmed from the measurements of the sky lines. The two-dimensional frames were sky subtracted using BACKGROUND. One-dimensional spectra were obtained integrating the signal along the slit using APS UM. The apertures were limited to regions where the signal intensity was above $1 \sigma$ of the sky noise.

The flux calibration was achieved using STANDARD SENSFUNC - CALIBRATE on spectra of the standard stars Feige 34, Hz 44 and Hiltner 600 (ESO) taken twice on each night. Cubic spline sensitivity functions of 20th order or higher were fit to the calibration spectra, allowing the transformation of the measured intensities into flux densities (erg s${ }^{-1} \mathrm{~cm}^{-2} \AA^{-1}$ ), including the atmospheric extinction correction. However, because during an exposure taken in the "drift-scan" mode the fraction of light collected by the slit changes with time, an absolute calibration of our science spectra cannot be achieved. Thus all spectra were normalized to their intensity at $\lambda=5500 \AA$. The spectrophotomectric standards were instead used to calibrate the absolute response of the system as a function of wavelength. The uncertainty of this measurement over the whole spectral region resulted within $15 \%$, as derived comparing the spectra of four galaxies taken in this work with the corresponding ones by K92 (see Sect. 2.3).

Three template spectra with high signal-to-noise ratio were selected for being representative of absorption-line objects, of weak emission-line objects and of strong emission-line objects respectively. They were shifted to their rest frame wavelength. All the remaining spectra were cross-correlated with one of these template spectra using FXCOR, thus providing their relative redshift. All spectra were shifted to the rest frame wavelength using $D O P C O R$ to better than $1 \AA$ and finally they were normalized to their intensity determined in the interval 5400-5600 ̊.

\subsection{Comparison with the K92 Atlas}

Four bright galaxies (NGC 1357, 1832, 3379 and 6181) were measured in common with K92 and their comparison is useful to assess the quality of our data. Figures 1 and 2 show the spectra of these objects as obtained by us (top spectra) and as given by K92 (bottom spectra). The ratio of the two measurements is shown in the bottom panel of each figure. The two sets of data are found in agreement within 15\% in the range 3800-6800 A.

\subsection{Synthetic and photometric color indices}

Synthesized spectroscopic colors $B-V$ and $B-R$ were obtained deconvolving the continua with the profiles of the $B, V$ and $R$ Johnson filters. Since the width of the $R$ filter (5500-7000 $\AA$ ) exceeds by $200 \AA$ in the red the domain covered by our spectra, $B-R$ is computed deconvolving the Bruzual \& Charlot (1993) population synthesis models fit to the observed spectra (see Paper I) with the $B$ and $R$ filter profiles. Figure 3 shows the comparison between the photometric colors $(B-V)_{T}$ and the $B-V$ color synthesized on the spectra for 312 galaxies. The two differ by 0.05 mag with an rms scatter of 0.15 mag.
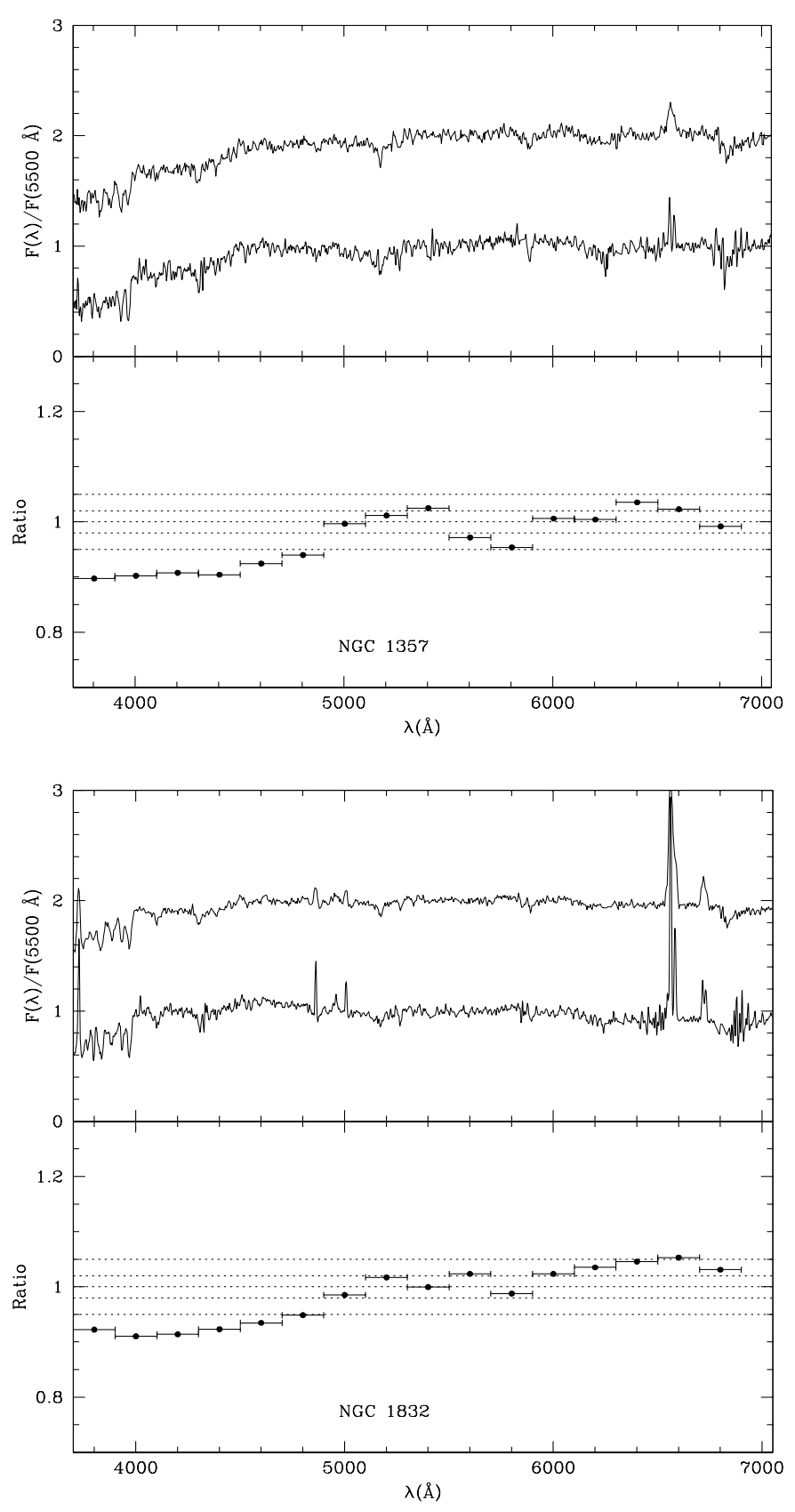

Fig. 1. Comparison between the spectra of NGC 1357 and 1832 obtained in this work and by K92. (top panels). The ratio of the two measurements is given in the bottom panels.

The comparison of the synthesized $B-R$ color from our spectra with $(B-V)_{T}$ color from photometry is given in Fig. 4. Unless otherwise specified, in this and in the following figures we plot the best fit linear regression obtained using the bysector method of Feigelson \& Babu (1992). The results of the linear regression analysis, including the uncertainties in their slope and zero point are summarized in Table 4.

\section{Line measurements}

Under visual inspection to the spectra we carried out a firstorder measurement to all lines, both in emission and in absorption, using S PLOT. This provided a list of fluxes and $E W \mathrm{~s}$ 

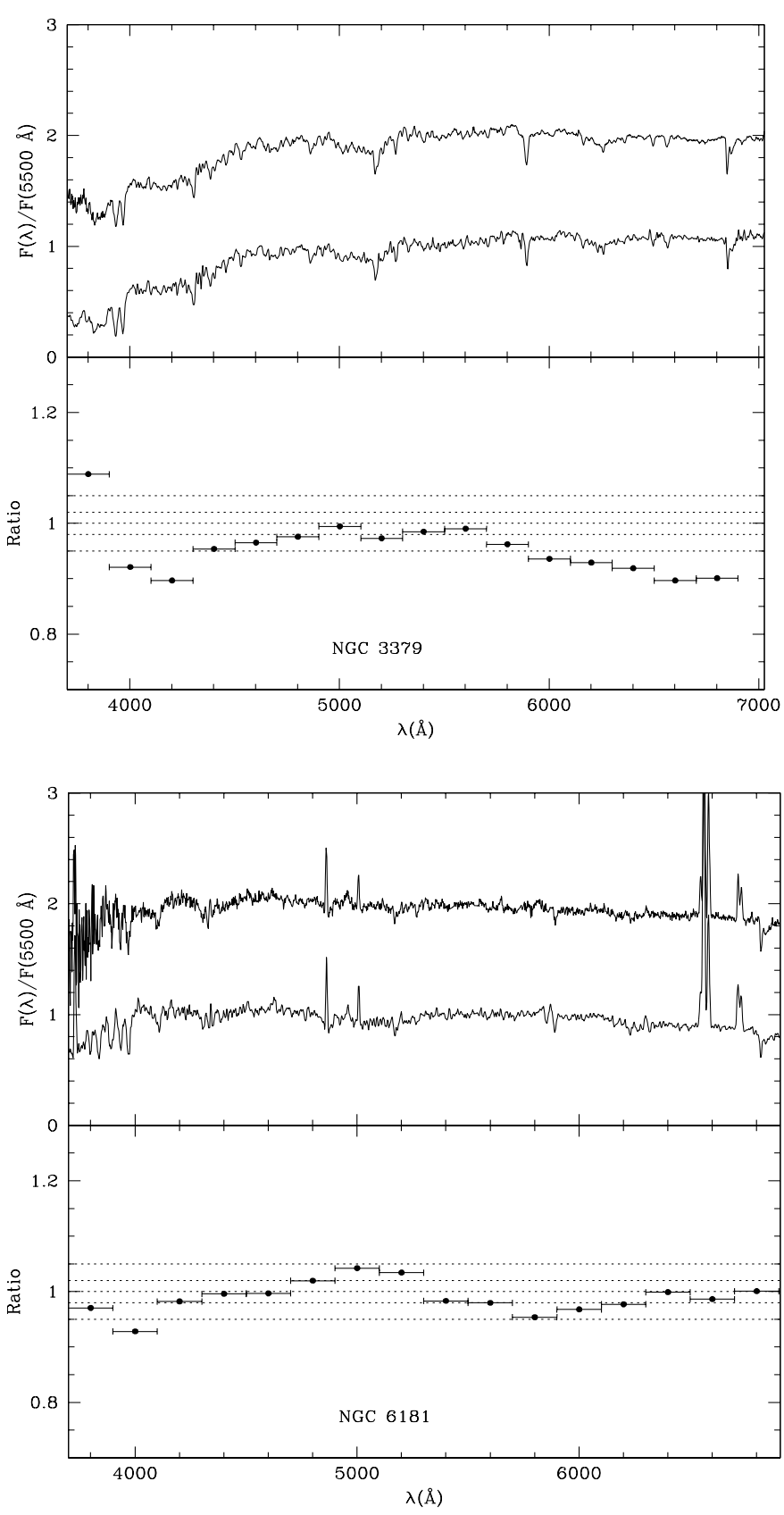

Fig. 2. Same as Fig. 1 for NGC 3379 and 6181.

with respect to a user defined continuum level. This preliminary measurement was then refined as described in the following sections.

\subsection{Deblending of $H_{\alpha}$ from [NII]}

$\mathrm{H}_{\alpha}$ ( $\left.\lambda 6563\right)$ is bracketed by the weaker [NII] doublet ([NII1] $\lambda 6548$ and [NII2] $\lambda 6584$ ). The three lines are clearly resolved in the OHP $(R=1000)$ spectra, thus for spectra taken at OHP the measurements of the individual lines is reliable. In the lower resolution $(R=500)$ spectra taken at ESO, Loiano and SPM the three lines are not well resolved, thus the deblending obtained with SPLOT is often inaccurate. In most of these cases we could only measure the global flux $T$

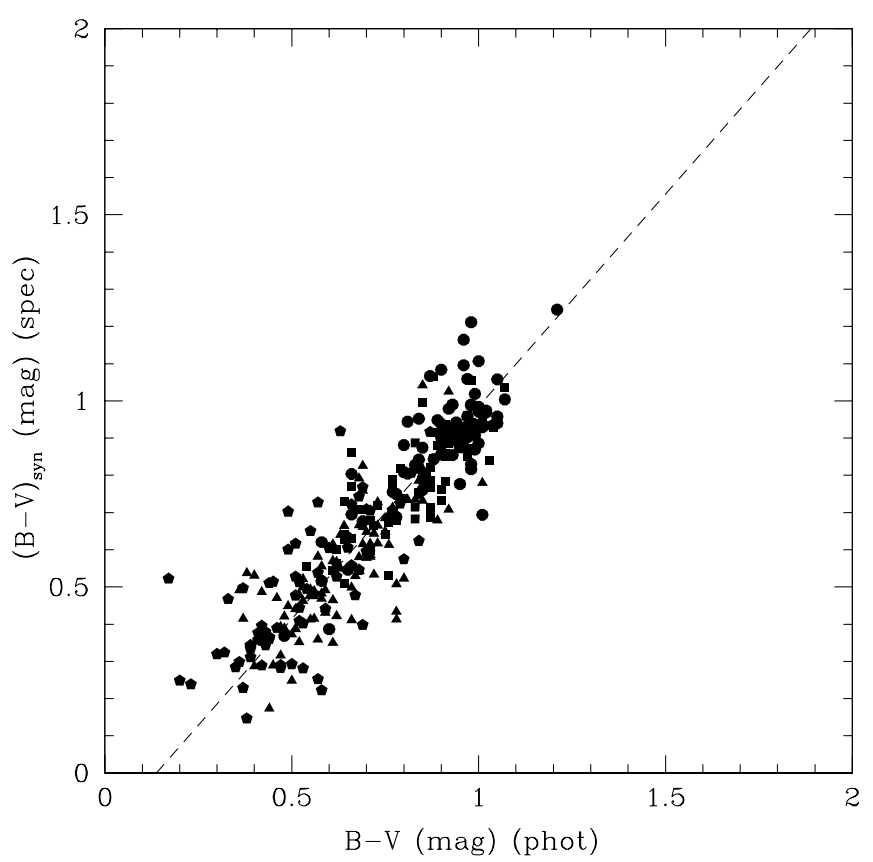

Fig. 3. Comparison of the synthesized $B-V$ color from our spectra and from photometry. Unless otherwise specified, in this and following figures $\mathrm{dE}-\mathrm{E}-\mathrm{SOa}$ are represented with circles; $\mathrm{Sa}-\mathrm{Sb}$ with squares; Sbc-Scd with triangles; Sd-BCD with pentagons. The dashed line represents the best linear fit to the data.

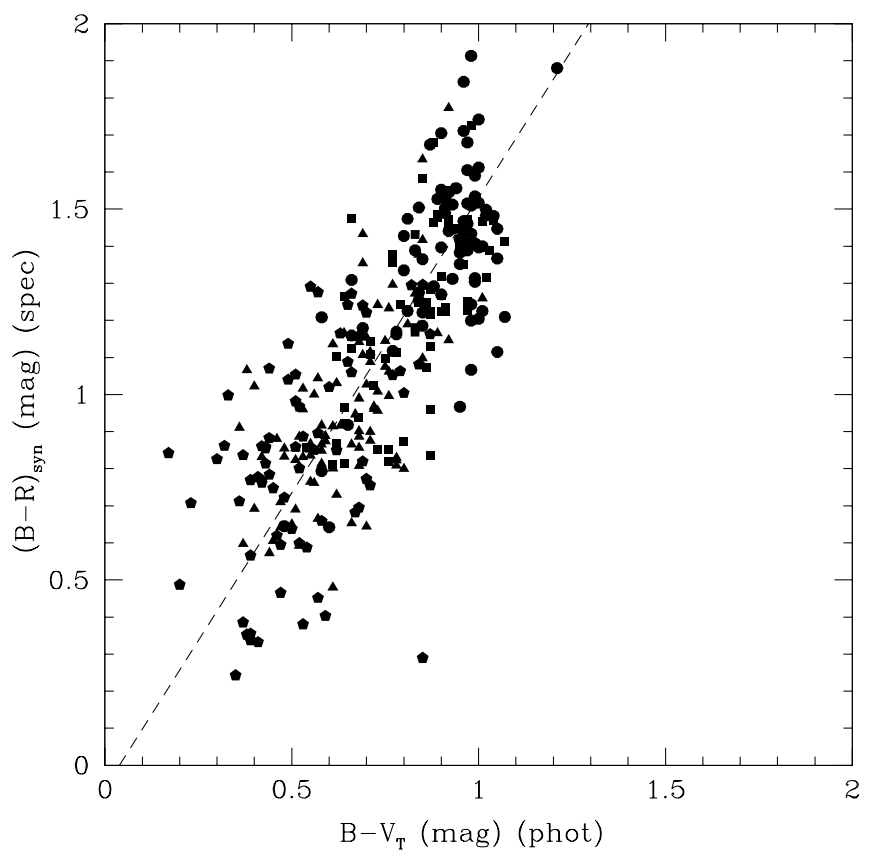

Fig. 4. Comparison of the synthesized $B-R$ color from our spectra with $(B-V)_{T}$ color from photometry. Same symbols as in Fig. 3. The dashed line represents the best linear fit to the data.

of the triplet $\mathrm{H}_{\alpha}+[\mathrm{NII} 1]+[\mathrm{NII} 2]$. To measure them individually we proceed as follows: we calibrate on the OHP spectra the empirical relation between $\left(([\mathrm{NII} 1]+[\mathrm{NII} 2]) / \mathrm{H}_{\alpha}\right)$ and the $V$-band luminosity shown in Fig. 5, which derives from the 
Table 4. The bysector linear regression analysis.

\begin{tabular}{lcc}
\hline \hline Regression & $R$ & see Fig. \\
\hline$(B-V)_{\text {syn }}=1.141 \pm 0.027 \times(B-V)_{T}-0.156 \pm 0.017$ & 0.86 & 3 \\
$(B-R)_{\text {syn }}=1.593 \pm 0.052 \times(B-V)_{T}-0.062 \pm 0.033$ & 0.78 & 4 \\
$\mathrm{H}_{\alpha}+[\mathrm{NII} 1]+[\mathrm{NII} 2]=0.575 \pm 0.052 \times \log \left(L_{V} / L_{\odot}\right)-5.922 \pm 0.206$ & 0.58 & 5 \\
$\mathrm{H}_{\alpha}+[\mathrm{NII} 1]+[\mathrm{NII} 2](s p)=1.189 \pm 0.054 \times \mathrm{H}_{\alpha}+[\mathrm{NII} 1]+[\mathrm{NII} 2](p h)-0.322 \pm 0.057$ & 0.75 & 7 \\
$\mathrm{Mg}_{2}=0.095 \pm 0.009 \times \log \left(L_{V} / L_{\odot}\right)-0.701 \pm 0.043$ & 0.71 & 14 \\
$\mathrm{NaD}=17.031 \pm 0.975 \times \mathrm{Mg}_{2}-0.660 \pm 0.221$ & 0.74 & 15 \\
$\mathrm{H}_{\beta}=-8.707 \pm 1.206 \times \mathrm{Mg}_{2}+4.045 \pm 0.218$ & -0.54 & 16 \\
$\mathrm{G}_{4300}=20.365 \pm 2.406 \times \mathrm{Mg}_{2}+0.227 \pm 0.446$ & 0.60 & 17 \\
$\Delta_{4000}=2.266 \pm 0.206 \times \mathrm{Mg}_{2}+0.040 \pm 0.040$ & 0.69 & 18 \\
\hline
\end{tabular}

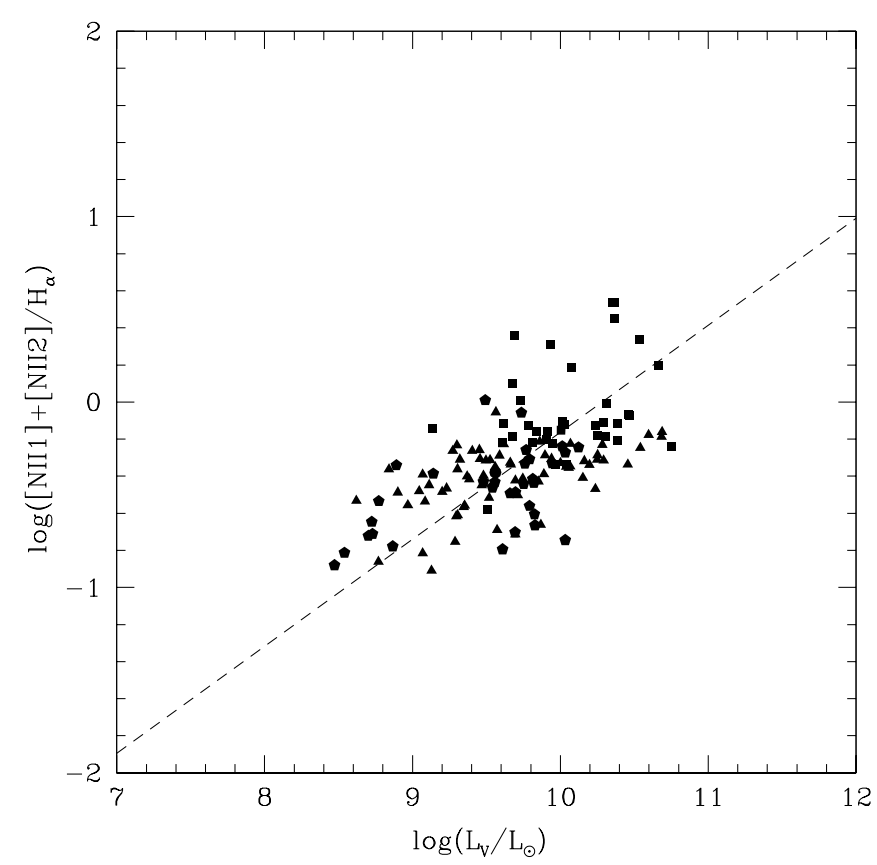

Fig. 5. $\log \left(([\mathrm{NII} 1]+[\mathrm{NII} 2]) / \mathrm{H}_{\alpha}\right)$ versus $V$-band luminosity for spectra taken at OHP. The dashed line represents the best linear fit to the data.

well known metallicity-luminosity relation (see Raimann et al. 2000). We find:

$\log \left(([\mathrm{NII} 1]+[\mathrm{NII} 2]) / \mathrm{H}_{\alpha}\right)=0.583\left(L_{V} / L_{\odot}\right)-5.996$,

which, coupled to [NII1] $=0.34$ [NII2] provides an estimate of each individual component [NII1], [NII2] and $\mathrm{H}_{\alpha}$.

We apply the above procedure only if [NII2] emission is detected, but not resolved from $\mathrm{H}_{\alpha}$, i.e. when the line separation is lower than the sum of the individual HWHM. Otherwise $\mathrm{H}_{\alpha}$ and [NII2] were measured individually and [NII1] was set as 0.34 [NII2].

The above procedure was checked a posteriori using 6 emission-line galaxies observed at ESO with both the low and the high resolution grisms. Figure 6 illustrates the consistency of the $E W$ of [NII2] and $\mathrm{H}_{\alpha}$ obtained on the low resolution spectra applying the deblending procedure with those directly measured on the HD spectra. The deblended measurements are found overestimated by $20 \%$ on average.

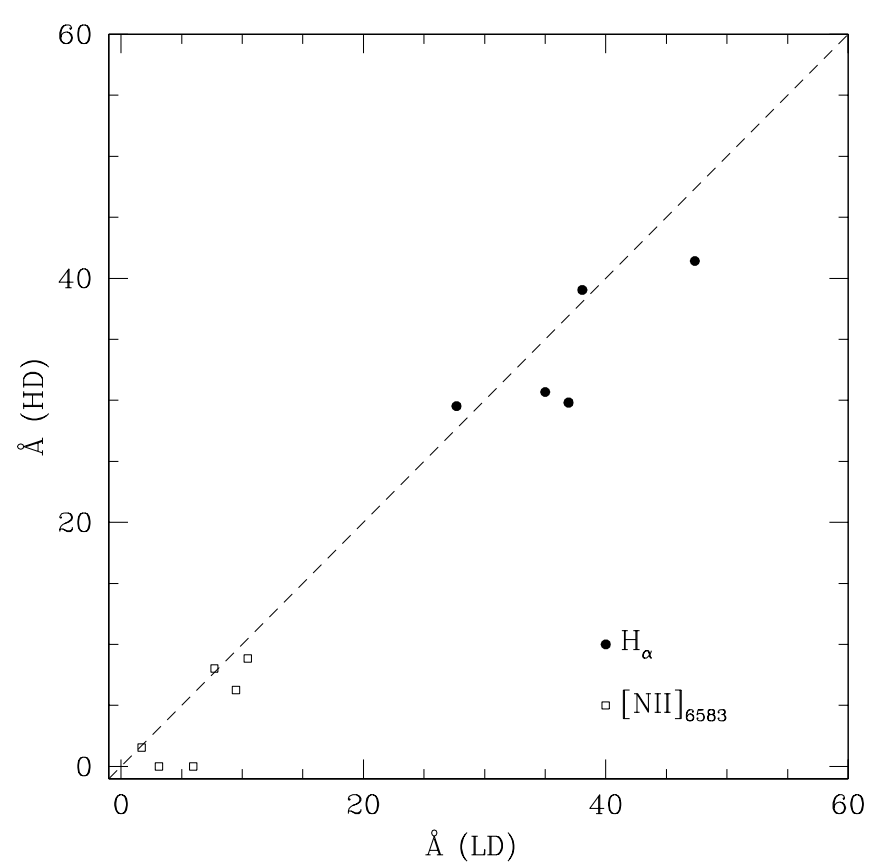

Fig. 6. Relation between [NII] and $\mathrm{H}_{\alpha} E W$ as measured in the High dispersion ESO spectra vs. the same quantities obtained on the Low dispersion spectra and deblended according to the procedure described in Sect. 3.1. The dashed line represents the one-to-one relation.

\subsection{Comparison with $H_{\alpha}$ from imaging}

Several (223) galaxies in our sample have their $\mathrm{H}_{\alpha}+[\mathrm{NII}]$ measured from imaging (Boselli \& Gavazzi 2002; Boselli et al. 2002; Gavazzi et al. 2002b). In spite of the different measuring techniques, the equivalent width derived from imaging and from our spectra are found within $0.31 \mathrm{dex}$ rms, as shown in Fig. 7. This confirms that the "drift-mode" spectroscopy is indeed representative of the entire galaxy, as claimed in the introduction.

\section{3. $[O I I] \lambda 3727$}

Due to low sensitivity in the blue of CARELEC, only $22 \%$ of the emission line objects $\left(E W \mathrm{H}_{\alpha}>0\right)$ observed at $\mathrm{OHP}$ have $[\mathrm{OII}](13727)$ detected, as opposed to $79 \%$ in ESO spectra whose rms noise at $4000 \AA$ is half that of OHP spectra. For the remaining OHP spectra we estimate $3 \times \sigma$ upper limits to the strength of [OII] as $3 \times \operatorname{rms}_{(3750-4050)} \times 7$, where $7 \AA$ is the mean $F W H M$ of the [OII] lines detected at OHP. 


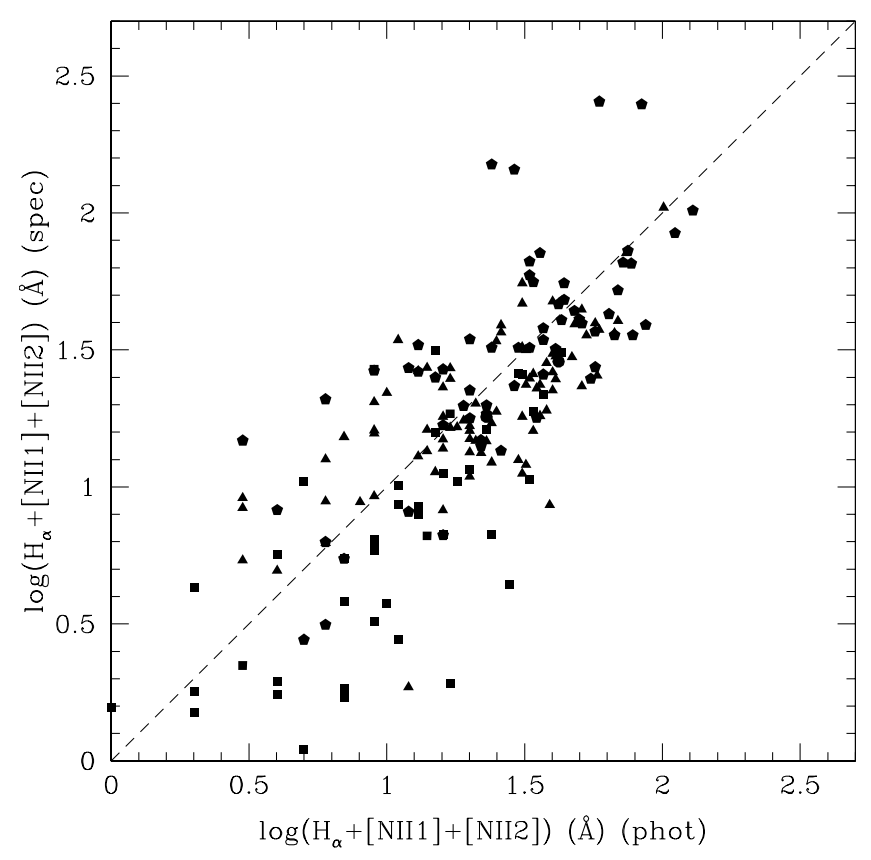

Fig. 7. Comparison of $\mathrm{H}_{\alpha}+[\mathrm{NII} 1]+[\mathrm{NII} 2] E W$ derived from imaging and from our spectra. Same symbols as in Fig. 3. The dashed line represents the one-to-one relation.

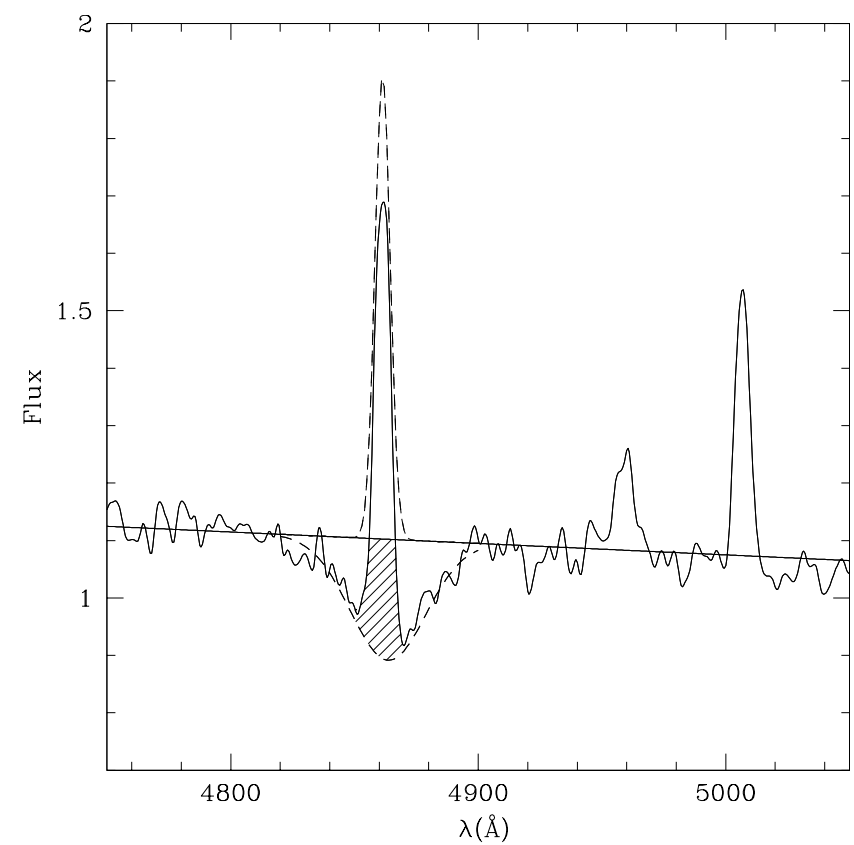

Fig. 8. Enlargement of the spectrum of VCC 25 to illustrate the deblending of $\mathrm{H}_{\beta}$ in emission from the underlying absorption. The observed $\mathrm{H}_{\beta}$ (continuum line) is deblended into a corrected emission and an absorption component (dotted lines). The shaded region represents the portion of the absorption line that is added to the emission line to obtain its correction.

\subsection{Correction for underlying absorption}

Most emission line galaxies show evidence for underlying absorption in correspondence to emission lines. In particular, out of 174 spectra where we could measure $\mathrm{H} \beta$ in emission, in 151 cases we detect significant $\mathrm{H} \beta$ in absorption, and in fewer cases $\mathrm{H} \gamma$ and $\mathrm{H} \delta$ as well. We deblended the underlying absorption from the emission lines using a multiple component fitting

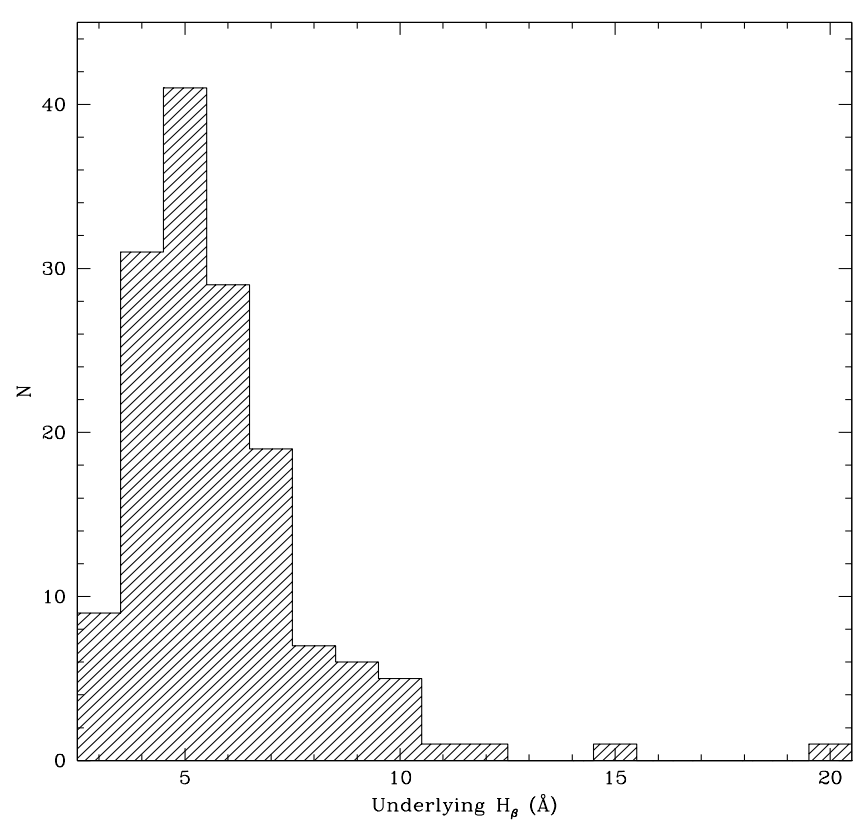

Fig. 9. Histogram of the underlying $E W$ in absorption at $\mathrm{H}_{\beta}$.

Table 5. Dereddening law relative to $\mathrm{H}_{\beta}$.

\begin{tabular}{ccc}
\hline \hline Line & $\lambda(\AA)$ & $\mathrm{f}(\lambda)-\mathrm{f}\left(\mathrm{H}_{\beta}\right)$ \\
\hline$[\mathrm{OII}]$ & 3727 & 0.31 \\
$\mathrm{H}_{\delta}$ & 4101 & 0.20 \\
$\mathrm{H}_{\gamma}$ & 4340 & 0.13 \\
$\mathrm{H}_{\beta}$ & 4861 & 0 \\
{$[\mathrm{OIII}]$} & 4958 & -0.02 \\
{$[\mathrm{OIII}]$} & 5007 & -0.03 \\
{$[\mathrm{NII}]$} & 6548 & -0.33 \\
$\mathrm{H}_{\alpha}$ & 6563 & -0.33 \\
{$[\mathrm{NII}]$} & 6584 & -0.34 \\
{$[\mathrm{SII}]$} & 6717 & -0.37 \\
{$[\mathrm{SII}]$} & 6731 & -0.37 \\
\hline
\end{tabular}

procedure written by us in the IRAF environment. To do so we measure the emission line and subtract it from the spectra. The resulting absorption line is also measured with respect to a reference continuum. These two measurements are used as first guess in a fitting algorithm which fits jointly the emission and absorption lines to the reference continuum (see Fig. 8). The value of the emission lines is given in Table 7, that of the underlying absorption in Table 8.

As shown in Fig. 9 the distribution of the underlying $\mathrm{H}_{\beta}$ is peaked at $5.7 \pm 1.9 \AA$, consistently with $\mathrm{K} 92$ who reported a mean underlying $\mathrm{H}_{\beta}$ of $5 \AA$.

For objects whose $\mathrm{H}_{\beta}$ was detected in emission but the deblending procedure was not applied (no absorption feature was evident) a mean additive correction for underlying absorption equal to -1.8 in flux and $-1.4 \AA$ in $E W$ was used. These values 


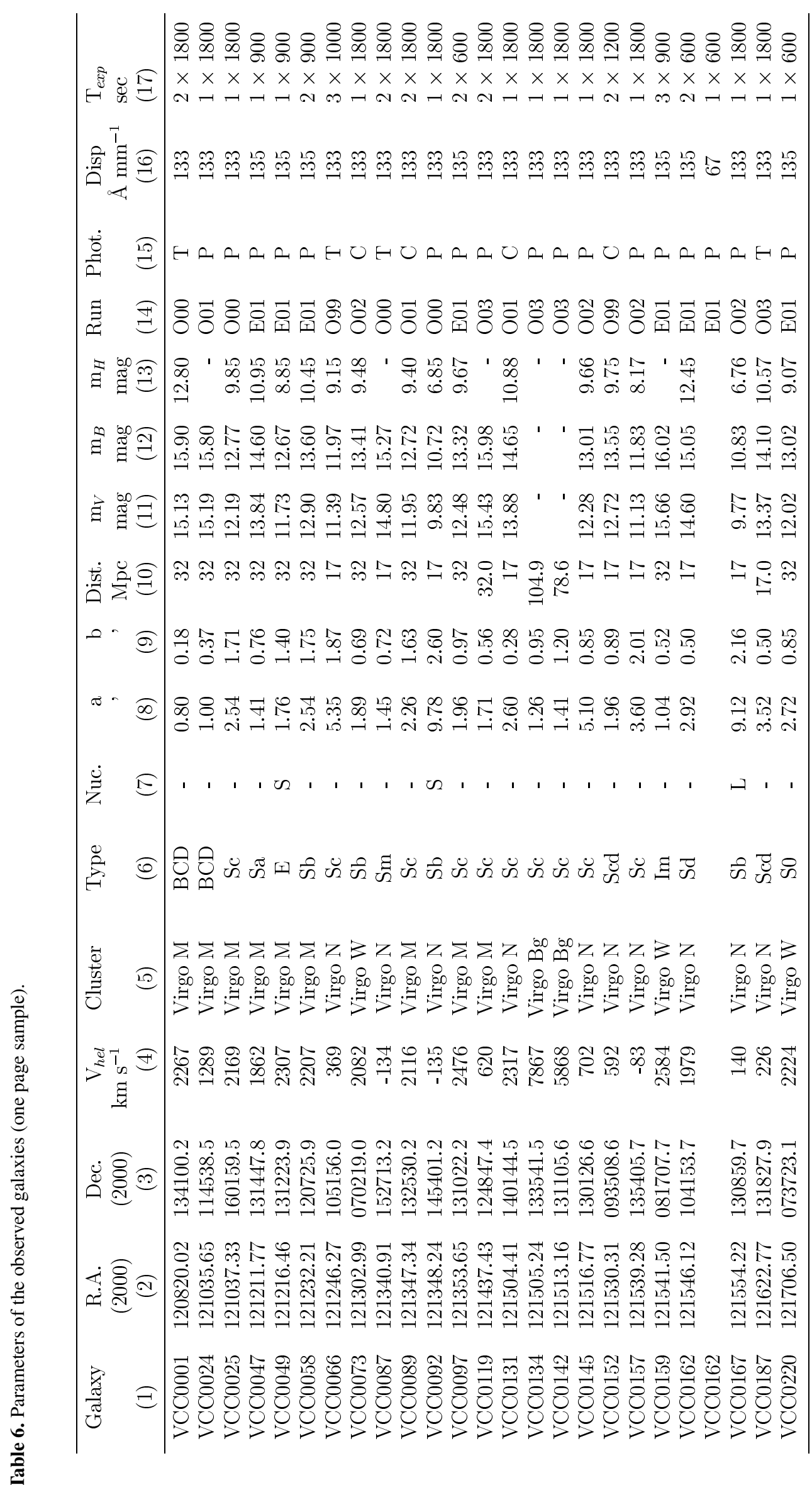




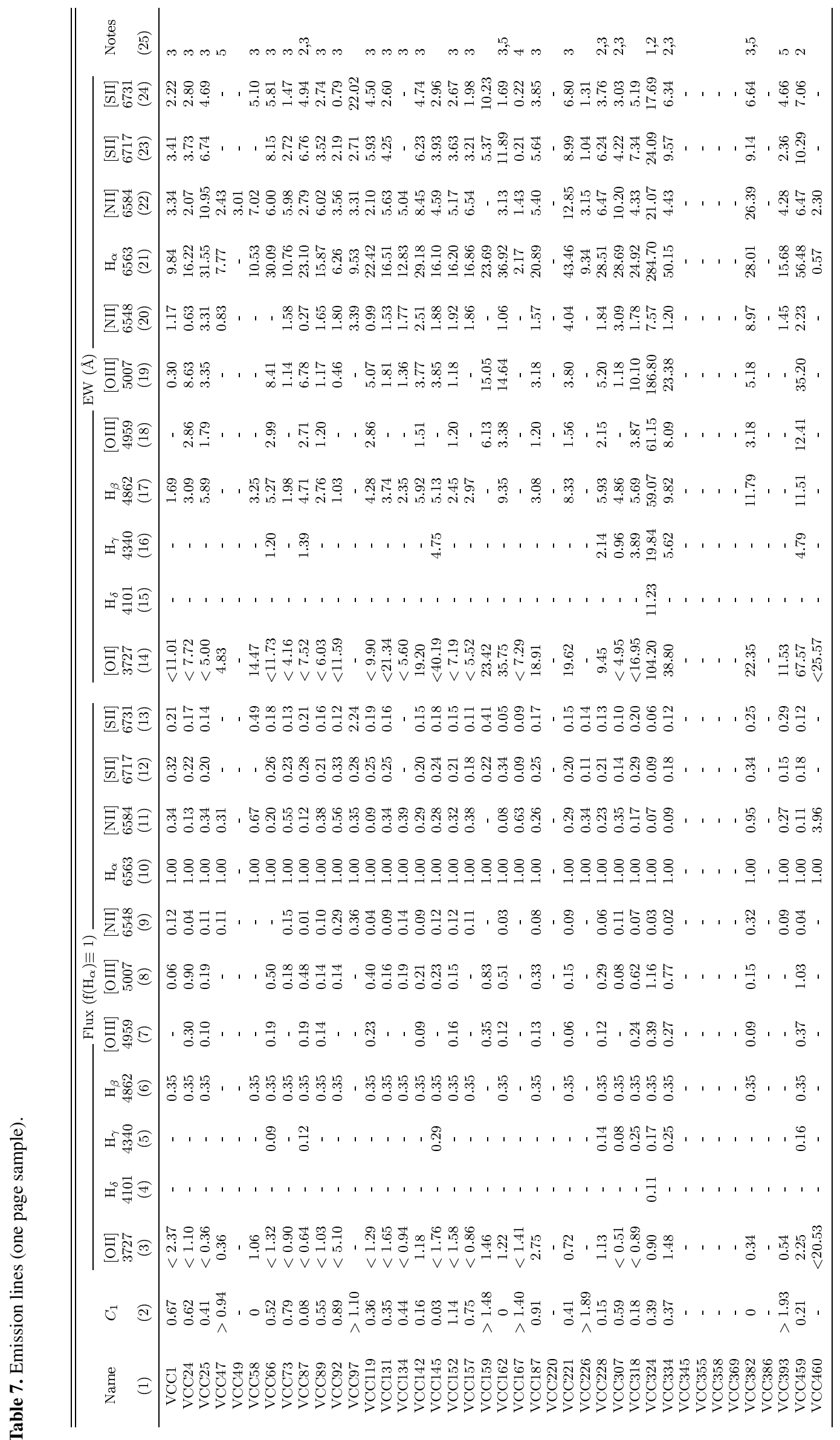


Table 8. Balmer absorption lines (one page sample).

\begin{tabular}{|c|c|c|c|c|c|c|c|c|c|c|c|}
\hline $\begin{array}{c}\text { Name } \\
(1)\end{array}$ & $\begin{array}{c}\mathrm{EWH}_{\delta} \\
(\AA) \\
(2)\end{array}$ & $\begin{array}{c}\mathrm{EWH}_{\gamma} \\
(\AA) \\
(3)\end{array}$ & $\begin{array}{c}\mathrm{EWH}_{\beta} \\
(\AA) \\
(4)\end{array}$ & $\begin{array}{c}\mathrm{EWH}_{\alpha} \\
(\AA) \\
(5)\end{array}$ & $\begin{array}{l}\text { Notes } \\
(6)\end{array}$ & $\begin{array}{c}\text { Name } \\
(1)\end{array}$ & $\begin{array}{c}\mathrm{EWH}_{\delta} \\
(\AA) \\
(2)\end{array}$ & $\begin{array}{c}\mathrm{EWH}_{\gamma} \\
(\AA) \\
(3)\end{array}$ & $\begin{array}{c}\mathrm{EWH}_{\beta} \\
(\AA) \\
(4)\end{array}$ & $\begin{array}{c}\mathrm{EWH}_{\alpha} \\
(\AA) \\
(5)\end{array}$ & $\begin{array}{l}\text { Notes } \\
(6)\end{array}$ \\
\hline $\mathrm{VCC} 1$ & - & - & -3.99 & - & 3 & VCC792 & -2.26 & - & - & - & \\
\hline VCC24 & -6.55 & -8.24 & -5.56 & - & 3 & VCC794 & - & - & - & - & \\
\hline $\mathrm{VCC} 25$ & -4.06 & - & -6.31 & - & 3 & VCC798 & - & - & -3.71 & -1.95 & \\
\hline VCC47 & - & - & -1.80 & - & & VCC801 & -4.96 & - & -8.26 & - & 3 \\
\hline VCC49 & - & - & -3.10 & -0.90 & & VCC809 & -3.24 & -1.95 & -8.25 & - & 3 \\
\hline VCC58 & -3.46 & -1.18 & -5.91 & - & 3 & VCC827 & - & - & -8.56 & - & 3 \\
\hline VCC66 & - & - & -5.41 & - & 3 & VCC828 & - & - & -2.96 & -1.70 & \\
\hline VCC73 & -3.26 & -1.48 & -4.27 & - & 3 & VCC836 & -5.33 & - & -3.28 & - & 1,3 \\
\hline VCC87 & -7.25 & -6.46 & -6.78 & - & 2,3 & VCC841 & -4.78 & -5.55 & - & - & 1,2 \\
\hline VCC89 & -2.86 & -2.84 & -4.95 & - & 3 & VCC848 & -6.99 & -4.86 & -5.45 & - & 3 \\
\hline VCC92 & - & - & -4.88 & - & 3 & VCC849 & -5.95 & - & -5.00 & - & 3 \\
\hline VCC97 & - & - & -1.70 & - & & VCC851 & -11.01 & - & -6.58 & - & 3 \\
\hline VCC119 & - & - & -2.86 & - & 3 & VCC857 & -3.72 & -1.27 & -2.59 & -2.05 & \\
\hline VCC131 & - & - & -7.85 & - & 3 & VCC865 & -4.54 & - & -6.99 & - & 3 \\
\hline VCC134 & -2.77 & - & -6.12 & - & 3 & VCC873 & -2.98 & -2.46 & -5.80 & - & 3 \\
\hline VCC142 & -3.52 & -1.28 & -5.42 & - & 3 & VCC874 & -3.45 & -3.37 & -8.07 & - & 3 \\
\hline VCC145 & - & - & - & - & & VCC905 & -3.44 & - & -3.69 & - & 3 \\
\hline VCC152 & -7.68 & -3.64 & -6.10 & - & 3 & VCC912 & -4.60 & - & -4.33 & - & 3 \\
\hline VCC157 & -5.06 & -2.08 & -5.52 & - & 3 & VCC921 & -2.62 & - & -4.40 & - & 3 \\
\hline VCC159 & -5.61 & -3.07 & - & - & & VCC938 & -2.81 & -2.14 & -5.92 & - & 3 \\
\hline VCC162 & -4.29 & -9.86 & -9.52 & - & 3 & VCC939 & - & - & -3.91 & - & 3 \\
\hline VCC167 & - & - & - & -2.75 & 4 & VCC945 & -5.88 & -6.29 & - & - & \\
\hline VCC187 & -6.98 & -3.77 & -5.16 & - & 3 & VCC950 & - & - & - & - & \\
\hline VCC220 & - & - & - & -0.73 & & VCC951 & -3.75 & -2.52 & -4.34 & -3.80 & \\
\hline VCC221 & -10.14 & - & -6.23 & - & 3 & VCC957 & -4.12 & -0.78 & -6.75 & - & 3 \\
\hline VCC226 & -2.62 & -2.08 & - & - & & VCC958 & -3.55 & -1.21 & - & - & \\
\hline VCC228 & -3.54 & -5.24 & -4.53 & - & 2,3 & VCC971 & -2.37 & - & -5.18 & - & 3 \\
\hline VCC307 & -3.92 & -3.18 & -5.36 & - & 2,3 & VCC973 & - & - & -1.93 & - & 3 \\
\hline VCC318 & -2.37 & - & - & - & & VCC975 & -6.86 & - & - & - & \\
\hline VCC324 & -6.90 & -5.29 & - & - & 1,2 & VCC979 & -7.26 & -4.46 & -5.68 & - & 3 \\
\hline VCC334 & - & -6.87 & -6.63 & - & 2,3 & VCC980 & -3.82 & - & -5.52 & - & 3 \\
\hline VCC345 & - & - & - & -1.08 & & VCC984 & - & - & -3.71 & -1.25 & \\
\hline VCC355 & -1.29 & -0.71 & -1.71 & -0.63 & & VCC995 & -6.33 & - & - & - & \\
\hline VCC358 & -1.39 & -1.11 & -2.29 & -1.86 & & VCC1002 & -4.47 & -3.78 & -4.27 & - & 3 \\
\hline VCC369 & - & - & - & -1.30 & & VCC1003 & - & - & -1.15 & -0.89 & 4 \\
\hline VCC382 & -5.31 & - & -12.13 & - & 3 & VCC1010 & - & - & -3.80 & -1.74 & \\
\hline VCC386 & - & - & - & - & & VCC1018 & -4.79 & -0.78 & -10.30 & - & 3 \\
\hline VCC393 & -7.35 & - & - & - & & VCC1028 & -2.77 & -5.57 & -2.78 & -2.44 & \\
\hline VCC459 & -1.64 & -3.80 & - & - & 2 & VCC1030 & - & - & -3.09 & -1.45 & \\
\hline VCC460 & -4.80 & - & - & - & & VCC1036 & -5.34 & -2.12 & -3.55 & -1.55 & \\
\hline VCC465 & - & -1.79 & -5.87 & - & 2,3 & VCC1043 & - & - & -2.80 & - & \\
\hline VCC483 & - & - & -4.51 & - & & VCC1047 & -1.65 & - & -3.64 & -1.47 & \\
\hline VCC491 & -7.02 & -3.54 & -6.93 & - & $1,2,3$ & VCC1062 & - & - & - & -1.00 & \\
\hline VCC497 & - & - & - & - & & VCC1068 & - & - & -2.58 & - & \\
\hline VCC508 & -2.26 & - & -4.60 & - & 3 & VCC1073 & - & - & -3.25 & - & \\
\hline VCC522 & - & - & - & -1.84 & & VCC1086 & -3.92 & - & - & - & \\
\hline VCC523 & - & - & - & - & & VCC1091 & - & - & -5.41 & - & 3 \\
\hline VCC534 & - & - & -4.44 & - & & VCC1107 & - & - & - & - & \\
\hline VCC538 & - & - & -2.64 & - & & VCC1110 & -3.15 & -1.00 & -2.00 & -0.63 & 4 \\
\hline VCC552 & -13.01 & -5.45 & -6.35 & - & 2,3 & VCC1118 & - & - & -4.96 & - & 3 \\
\hline VCC559 & -3.06 & -2.16 & - & -2.92 & 4 & VCC1125 & - & - & -3.69 & -1.39 & \\
\hline VCC562 & - & - & - & - & & VCC1145 & -1.30 & -1.65 & -6.91 & - & \\
\hline VCC596 & - & - & - & - & & VCC1146 & -1.40 & - & -2.98 & -1.76 & \\
\hline VCC630 & -3.78 & - & - & - & & VCC1154 & - & - & - & -1.24 & \\
\hline VCC634 & - & - & - & -3.16 & & VCC1158 & -1.66 & -0.71 & -4.38 & -2.18 & \\
\hline VCC636 & - & - & - & -3.39 & & VCC1189 & - & -2.24 & -4.85 & - & 2,3 \\
\hline VCC655 & - & - & -4.27 & - & 3 & VCC1192 & - & - & -3.45 & -2.24 & \\
\hline VCC656 & -2.03 & -1.05 & -3.70 & -1.70 & 4 & VCC1193 & -3.47 & - & -6.61 & - & 3 \\
\hline VCC664 & -4.15 & -7.79 & -6.73 & - & $1,2,3$ & VCC1196 & -2.09 & - & -4.04 & -1.81 & \\
\hline VCC667 & -6.45 & - & -6.15 & - & 3 & VCC1200 & -9.28 & -8.78 & -4.57 & - & \\
\hline VCC685 & - & - & -3.14 & -1.75 & & VCC1203 & - & - & -3.74 & -2.44 & \\
\hline VCC688 & -3.40 & -1.72 & -5.09 & - & 3 & VCC1205 & -5.18 & -0.81 & -6.70 & - & 3 \\
\hline VCC692 & -4.80 & - & -5.39 & - & 3 & VCC1217 & -6.49 & -6.13 & -9.65 & - & \\
\hline VCC699 & -3.93 & - & -11.27 & - & 3 & VCC1226 & - & - & -2.84 & -1.50 & \\
\hline VCC713 & - & - & -2.60 & - & & VCC1231 & - & - & - & -1.53 & \\
\hline VCC731 & - & - & -3.13 & -1.56 & & VCC1242 & -1.54 & - & -2.97 & -1.50 & \\
\hline VCC758 & - & - & -4.20 & - & & VCC1253 & - & - & - & - & \\
\hline VCC759 & - & - & - & -1.38 & & VCC1254 & - & - & -2.98 & -1.25 & \\
\hline VCC762 & -1.97 & - & -1.44 & -2.93 & & VCC1283 & - & - & -1.58 & -1.92 & \\
\hline VCC781 & -2.89 & -1.29 & -3.48 & - & & VCC1290 & -4.03 & -1.62 & -10.36 & - & 3 \\
\hline VCC787 & -4.97 & -7.13 & -9.99 & - & 2,3 & VCC1297 & - & - & -3.30 & -1.74 & \\
\hline
\end{tabular}




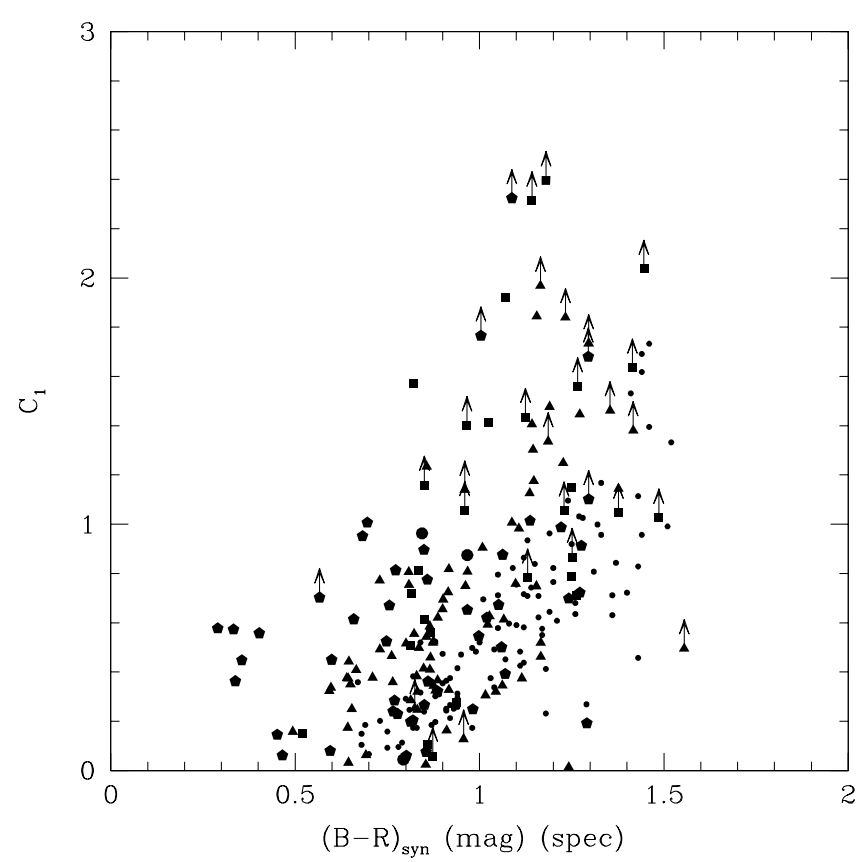

Fig. 10. $C_{1}$ versus $(B-R)_{\text {syn }}$ (excluding Seyfert objects). Same symbols as in Fig. 3 with the addition of galaxies observed by Jansen et al. (2000) (small dots).

correspond to the fraction of the (broader) absorption feature that lies under the emission feature.

No mean correction was applied to other lines except $\mathrm{H}_{\beta}$.

\subsection{The Balmer decrement $\left(C_{1}\right)$}

From $\mathrm{H}_{\beta}$ corrected for underlying absorption (Sect. 3.4) and $\mathrm{H}_{\alpha}$ corrected for deblending from [NII] (Sect. 3.1) we evaluate the Balmer decrement:

$C_{1}=\left(\log \left(\mathrm{H}_{\alpha} / \mathrm{H}_{\beta}\right)_{\text {theor }}-\log \left(\mathrm{H}_{\alpha} / \mathrm{H}_{\beta}\right)_{\text {obs }}\right) /\left(f\left(\mathrm{H}_{\alpha}\right)-f\left(\mathrm{H}_{\beta}\right)\right)$

(in the current notation: $\mathrm{A}\left(\mathrm{H}_{\beta}\right)=2.5^{*} C_{1}$ ).

The ratio $\log \left(\mathrm{H}_{\alpha} / \mathrm{H}_{\beta}\right)$ theor depends on the electron density and on the gas temperature. Assuming $T=10000 \mathrm{~K}$ and $n=$ $100 \mathrm{e} / \mathrm{cm}^{3}$, as in Osterbrock (1989) case B, $\left(\mathrm{H}_{\alpha} / \mathrm{H}_{\beta}\right)_{\text {theor }}=$ 2.86 holds.

The corrected line fluxes are derived, relative to $\mathrm{H}_{\beta}$, using $C_{1}$ and the reddening function $f(\lambda)$ of Lequeux et al. (1979) (see Table 3.5) based on the extinction law of Whitford (1958) ${ }^{4}$.

When $E W \mathrm{H}_{\alpha}>1 \AA$ but $\mathrm{H}_{\beta}$ is undetected we derive a $3 \times \sigma$ lower limit to $C_{1}$ using (Buat et al. 2002):

$\mathrm{H}_{\beta}<3 \times \mathrm{rms}_{(4500-4800)} \times \mathrm{H}_{\alpha} H W H M$

assuming that $\mathrm{H}_{\alpha}$ and $\mathrm{H}_{\beta}$ have similar $H W H M$ (Half Width Half Maximum).

${ }^{4}$ We have compared the $f(\lambda)$ of Lequeux et al. (1979) with the derivation of Cardelli et al. (1989), which assumes the extinction law of Seaton (1979) and of Savage \& Mathis (1979). The two functions are in agreement (in the optical range of our interest): differences are $\leq 0.03$ from [OII] to $\mathrm{H} \alpha$ (included); 0.05 at [SII].
Figure 10 shows the obtained $C_{1}$ as a function of the synthetic $B-R$ color index for our objects (coded according to the morphological type) and for galaxies observed by Jansen et al. (2000) and analyzed by Stasinska \& Sodre (2001). Our data confirm the positive correlation between the two quantities: i.e. increasing $C_{1}$ with increasing $B-R$. However the dispersion appears higher and the relation steeper than in Stasinska \& Sodre (2001). Notice that these authors did not measure the underlying absorption at $\mathrm{H}_{\beta}$.

\section{Results}

The 333 (rest-framed and normalized) spectra obtained in this work are illustrated in Fig. 19.

The ESO spectra are given in the range 3600-6800 A. The OHP spectra, noisier in the blue, were resampled with a step of $5 \AA$ and are given from 3850 to $6800 \AA$, unless the strength of the [OII] line was higher than 3 times the noise determined locally near the line (see Sect. 3.3). The flux scale of Fig. 19 is given in three intervals: $0.2-2 ; 0.2-5,0.2-15$ according to the intensity of the brightest lines. The additional 6 high dispersion spectra obtained at ESO are given in the last page of Fig. 19.

All spectra presented in Fig. 19 are available in the FITS format at the WEB site GOLDMine (http://goldmine.mib.infn.it/).

\subsection{Emission lines}

The (corrected) emission lines parameters are listed in Table 7:

Column 1: galaxy identification.

Column 2: Balmer decrement $C_{1}$ (or lower limit).

Columns 3-13: line intensities corrected for Balmer decrement, normalized to $\mathrm{H}_{\alpha}$.

Column 3: [OII] ( 33727) (or upper limit).

Column 4: $\mathrm{H}_{\delta}(\lambda 4101)$.

Column 5: $\mathrm{H}_{\gamma}($ (24340).

column 6: $\mathrm{H}_{\beta}(\lambda 4861)$.

Column 7: [OIII] (ג4959).

Column 8: [OIII] ( $\lambda 5007)$.

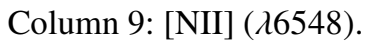

Column 10: $\mathrm{H}_{\alpha}(\lambda 6563)$.

Column 11: [NII] (ג6584).

Column 12: [SII] ( 26717$)$.

Column 13: [SII] (26731).

Columns 14-24: equivalent widths $(\AA)$.

Column 14: [OII] ( 23727$)$ (or upper limit).

Column 15: $\mathrm{H}_{\delta}(\lambda 4101)$.

Column 16: $\mathrm{H}_{\gamma}(\lambda 4340)$.

Column 17: $\mathrm{H}_{\beta}(\lambda 4861)$.

Column 18: [OIII] (ג4959).

Column 19: [OIII] ( $\lambda 5007)$.

Column 20: [NII] ( $\lambda 6548)$.

Column 21: $\mathrm{H}_{\alpha}(\lambda 6563)$.

Column 22: [NII] ( $\lambda 6584)$.

Column 23: [SII] (26717).

Column 24: [SII] (26731).

Column 25: notes. 
Table 9. Absorption indices.

\begin{tabular}{|c|c|c|c|c|c|c|c|c|c|c|c|}
\hline $\begin{array}{l}\text { Name } \\
(1)\end{array}$ & $\begin{array}{c}\Delta_{4000} \\
\operatorname{mag} \\
(2)\end{array}$ & $\begin{array}{c}\mathrm{EW} G_{4300} \\
\AA \\
(3)\end{array}$ & $\begin{array}{c}\mathrm{EWH}_{\beta} \\
\AA \\
(4)\end{array}$ & $\begin{array}{l}M g_{2} \\
\operatorname{mag} \\
(5)\end{array}$ & $\begin{array}{c}\text { EW NaD } \\
\AA \\
(6)\end{array}$ & $\begin{array}{l}\text { Name } \\
(1)\end{array}$ & $\begin{array}{c}\Delta_{4000} \\
\operatorname{mag} \\
(2)\end{array}$ & $\begin{array}{c}\mathrm{EW} G_{4300} \\
\AA \\
(3)\end{array}$ & $\begin{array}{c}\mathrm{EWH}_{\beta} \\
\AA \\
(4)\end{array}$ & $\begin{array}{l}M g_{2} \\
\operatorname{mag} \\
(5)\end{array}$ & $\begin{array}{c}\text { EW NaD } \\
\AA \\
(6)\end{array}$ \\
\hline VCC49 & 0.55 & 5.34 & 1.82 & 0.22 & 2.90 & VCC1348 & 0.75 & 1.52 & 2.24 & 0.21 & 1.99 \\
\hline VCC 220 & 0.60 & 6.94 & 1.82 & 0.27 & 2.66 & VCC1368 & 0.50 & 5.02 & 2.60 & 0.18 & 1.39 \\
\hline VCC345 & 0.50 & 4.98 & 1.70 & 0.31 & 5.06 & VCC1491 & 0.64 & 2.62 & 2.21 & 0.14 & 2.54 \\
\hline VCC 355 & 0.61 & 5.66 & 1.42 & 0.28 & 2.55 & VCC1499 & 0.25 & 1.21 & 4.99 & 0.07 & 0.62 \\
\hline VCC369 & 0.70 & 5.22 & 1.94 & 0.29 & 3.35 & VCC1619 & 0.55 & 4.67 & 1.90 & 0.20 & 3.17 \\
\hline VCC523 & 0.18 & 3.52 & 2.62 & 0.14 & 0.52 & VCC1630 & 0.59 & 5.13 & 2.00 & 0.25 & 2.98 \\
\hline VCC538 & 0.37 & 4.01 & 2.65 & 0.11 & 3.40 & VCC1632 & 0.36 & 5.68 & 1.70 & 0.28 & 4.77 \\
\hline VCC634 & 0.27 & - & 1.27 & 0.08 & - & VCC1683 & 0.16 & 3.66 & 3.44 & 0.06 & 0.82 \\
\hline VCC636 & 0.29 & 2.21 & 1.41 & 0.17 & 0.99 & VCC1869 & 0.42 & 5.43 & 1.86 & 0.25 & 3.55 \\
\hline VCC685 & 0.58 & 5.17 & 1.65 & 0.30 & 4.40 & VCC1903 & 0.39 & 4.64 & 1.91 & 0.28 & 5.00 \\
\hline VCC731 & 0.58 & 5.78 & 1.68 & 0.31 & 3.89 & VCC1938 & 0.59 & 5.23 & 2.23 & 0.28 & 4.88 \\
\hline VCC758 & 0.55 & 4.41 & 3.44 & 0.19 & 3.27 & VCC1978 & 0.49 & 5.55 & 1.54 & 0.33 & 5.75 \\
\hline VCC759 & 0.39 & 5.04 & 1.67 & 0.25 & 3.41 & VCC2000 & 0.42 & 5.31 & 1.72 & 0.25 & 3.02 \\
\hline VCC762 & 0.48 & 4.76 & 1.25 & 0.18 & 0.41 & VCC2087 & 0.59 & 5.23 & 3.14 & 0.19 & - \\
\hline VCC781 & 0.28 & 5.45 & 3.17 & 0.09 & 3.98 & VCC2092 & 0.48 & 5.63 & 2.02 & 0.26 & 3.65 \\
\hline VCC794 & 0.34 & 0.24 & 2.47 & 0.16 & 2.26 & VCC2095 & 0.60 & 5.55 & 1.65 & 0.27 & 3.40 \\
\hline VCC798 & 0.59 & 4.12 & 2.37 & 0.22 & 3.43 & Z14034 & 0.15 & 2.69 & - & 0.09 & 1.92 \\
\hline VCC 828 & 0.56 & 5.74 & 1.82 & 0.24 & 3.28 & Z69058 & 0.27 & 4.20 & 2.73 & 0.14 & 2.46 \\
\hline VCC951 & 0.37 & 3.28 & 2.45 & 0.10 & 1.79 & Z97088 & 0.60 & 0.90 & 2.45 & 0.16 & 2.87 \\
\hline VCC1003 & 0.59 & 5.28 & 1.39 & 0.27 & 3.51 & Z97097 & 0.75 & 5.51 & 2.24 & 0.24 & 2.81 \\
\hline VCC1010 & 0.58 & 4.61 & 2.12 & 0.16 & 1.85 & Z97123 & 0.22 & 0.68 & - & 0.08 & 1.77 \\
\hline VCC1028 & 0.12 & - & 2.34 & 0.02 & 0.38 & Z97125 & 0.22 & 1.64 & - & 0.15 & 3.26 \\
\hline VCC1030 & 0.51 & 4.93 & 1.90 & 0.24 & 3.38 & Z100011 & 0.55 & 4.21 & 2.34 & 0.17 & 3.23 \\
\hline VCC1036 & 0.45 & 5.23 & 2.93 & 0.16 & 1.05 & Z119024 & 0.65 & 5.25 & 2.11 & 0.25 & 3.04 \\
\hline VCC1062 & 1.11 & 5.47 & 1.96 & 0.31 & 5.06 & Z119031 & 0.76 & 6.97 & 0.76 & 0.29 & 4.60 \\
\hline VCC1068 & 0.65 & 6.83 & 2.80 & 0.28 & 5.72 & Z119048 & 0.59 & 6.41 & 1.53 & 0.30 & 5.07 \\
\hline VCC1073 & 0.54 & 3.18 & 1.76 & 0.19 & 2.38 & Z119053 & 0.26 & 0.27 & - & 0.07 & 1.97 \\
\hline VCC1107 & 0.32 & 4.97 & 4.99 & 0.14 & 2.38 & Z119063 & 0.56 & 8.05 & 2.11 & 0.28 & 4.93 \\
\hline VCC1125 & 0.54 & 6.43 & 1.97 & 0.21 & 2.57 & Z119065 & 0.64 & 5.18 & 1.59 & 0.32 & 5.65 \\
\hline VCC1146 & 0.64 & 5.21 & 2.28 & 0.19 & 2.36 & Z119067 & 0.68 & 4.93 & 1.97 & 0.20 & 2.95 \\
\hline VCC1154 & 0.44 & 4.88 & 2.09 & 0.24 & 2.39 & Z119074 & 0.83 & 6.91 & 1.92 & 0.26 & 3.58 \\
\hline VCC1192 & 0.52 & 5.49 & 2.73 & 0.28 & 2.98 & Z119081 & 0.73 & 4.76 & 2.44 & 0.22 & 3.10 \\
\hline VCC1196 & 0.51 & 3.49 & 2.91 & 0.14 & 1.18 & Z160097 & 0.53 & 4.64 & 2.21 & 0.23 & 2.30 \\
\hline VCC1203 & 0.74 & 8.25 & 1.83 & 0.17 & 3.41 & Z160103 & 0.68 & 5.80 & 2.03 & 0.29 & 4.56 \\
\hline VCC1226 & 0.58 & 4.32 & 1.35 & 0.26 & 3.25 & Z160110 & 0.40 & 3.72 & 2.54 & 0.19 & 2.96 \\
\hline VCC1231 & 0.68 & 4.39 & 2.08 & 0.29 & 3.88 & Z160215 & 0.65 & 5.46 & 1.47 & 0.29 & 4.55 \\
\hline VCC1242 & 0.40 & 5.34 & 2.07 & 0.20 & 3.01 & Z160219 & 0.55 & 4.88 & 1.61 & 0.25 & 4.30 \\
\hline VCC1253 & 0.54 & 5.94 & 1.68 & 0.26 & 2.91 & Z160241 & 0.64 & 5.28 & 1.48 & 0.29 & 3.82 \\
\hline VCC1254 & 0.68 & 1.66 & 3.98 & 0.17 & 0.38 & Z160258 & 0.67 & 5.29 & 2.29 & 0.27 & 2.93 \\
\hline VCC1283 & 0.51 & 4.23 & 1.94 & 0.20 & 3.46 & $\mathrm{CCC} 45$ & 0.54 & 4.90 & 1.90 & 0.23 & 2.96 \\
\hline VCC1297 & 0.62 & 6.23 & 1.43 & 0.30 & 3.54 & CCC94 & 0.38 & 4.65 & 1.79 & 0.15 & 2.16 \\
\hline VCC1316 & 0.58 & 4.89 & 0.56 & 0.31 & 4.92 & CCC96 & 0.55 & 5.56 & 1.84 & 0.22 & 2.17 \\
\hline VCC1327 & 0.33 & 2.34 & 2.89 & 0.11 & 2.39 & CCC119 & 0.57 & 6.16 & 1.76 & 0.26 & 3.21 \\
\hline
\end{tabular}

\subsection{Balmer absorption lines}

Whenever an absorption feature is detected in correspondence of Balmer lines (either alone or deblended from emission, as discussed in Sect. 3.4) its $E W$ is listed in Table 8 as follows:

Column 1: galaxy identification.

Column 2: $\mathrm{H}_{\delta}(\lambda 4101)$.

Column 3: $\mathrm{H}_{\gamma}($ (24340).

Column 4: $\mathrm{H}_{\beta}(\lambda 4861)$.

Column 5: $\mathrm{H}_{\alpha}(\lambda 6563)$.

Column 6: notes.

Frequency distributions of the $E W \mathrm{~s}$ of the principal (emission

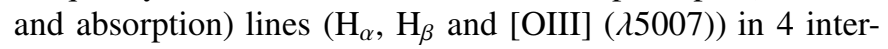
vals of Hubble type are given in Figs. 11-13 respectively. For each Hubble type interval, the total number of objects and the number of objects with a given measured line (either in emission or in absorption) is labeled in each panel. The continuum lines represent similar frequency distributions obtained with the Jansen et al. (2000) data. If one excludes the absorption lines (negative $E W$ ) that Jansen et al. (2000) did not measure, whereas they are included in our analysis, the two distributions appear consistent one another. In fact the probability that the two distributions are derived from the same parent populations is: $>62 \%$ for S0-Sab, $>97 \%$ for $\mathrm{Sb}-\mathrm{Sd}$ and $>57 \%$ for Sdm-BCD, as derived from the Kolmogorov-Smirnov test (dSO-E are excluded from this analysis due to the poor statistics). The implications of this finding is that, to the first order, galaxies in rich clusters do not have emission line properties dramatically different from isolated galaxies. Small differences, if any, require a more subtle analysis to be identified, which is postponed to Paper III.

\subsection{Absorption line indices}

The absorption lines indices are derived for early-type objects (dE-dS0-E-S0) and are listed in Table 9. They are derived according to the Lick system (Worthey et al. 1994) as the result of the integration in defined bands. Continua are determined on both sides of the line under measurement, and averaged. The line strength (or $E W$ ) is given as the difference between the 


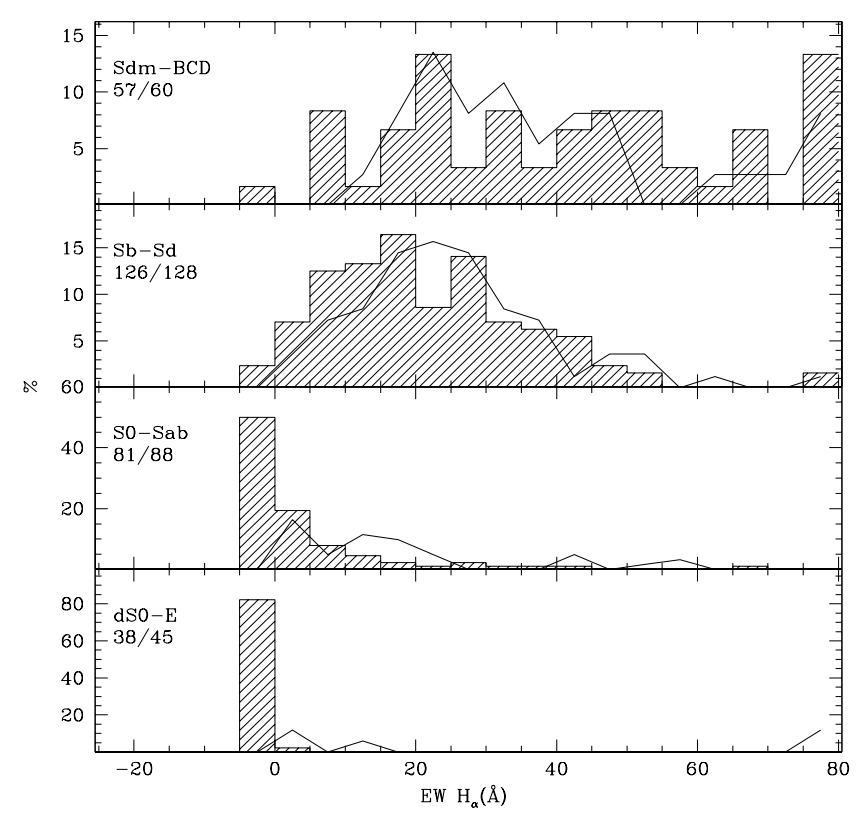

Fig. 11. Distribution of $\mathrm{H}_{\alpha} E W$ in four intervals of Hubble type. Positive $E W$ represent emission lines, negative values represent absorption lines. The continuum line represents the frequency distribution obtained with the Jansen et al. (2000) data.

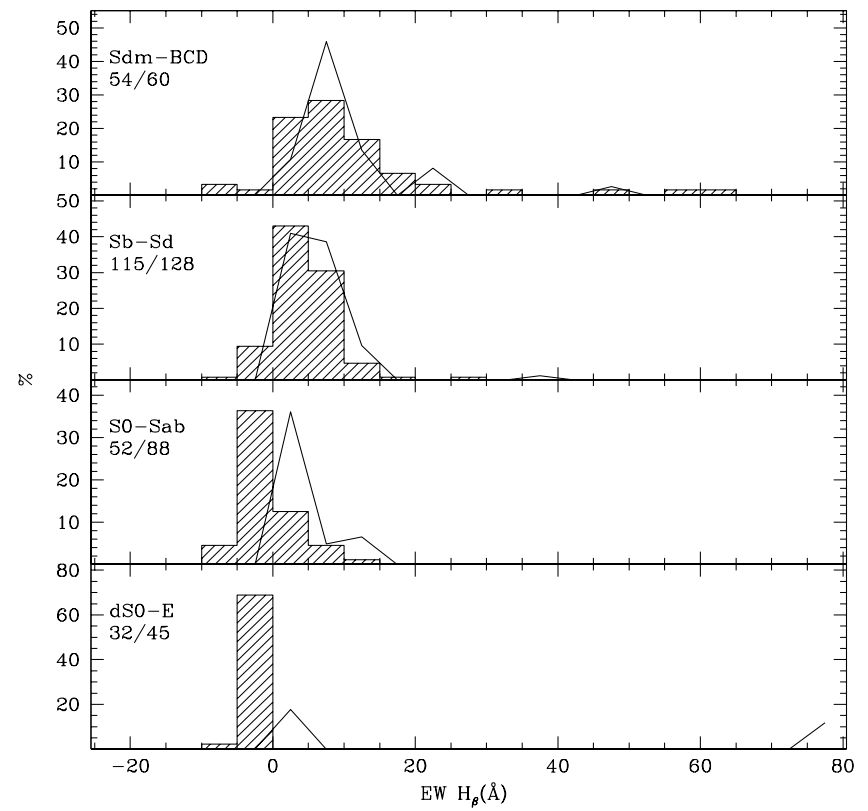

Fig. 12. Same as in Fig. 11 for $\mathrm{H}_{\beta} E W$.

integral in the interval containing the line and in the adjacent continuum ${ }^{5}$.

Table 9 is organized as follows:

Column 1: galaxy identification.

Column 2: calcium Break $\left(\Delta_{4000}\right)(\lambda 4000)$ in mag.

\footnotetext{
5 The integration has been carried in the Lick system, but the indices were not corrected using Lick standards. Notice that the $E W H \beta$ listed in Table 9 do not correspond with those in Table 8 for galaxies in common between the two Tables (e.g. elliptical galaxies with $\mathrm{H} \beta$ in absorption) because they are obtained with different measuring techniques.
}

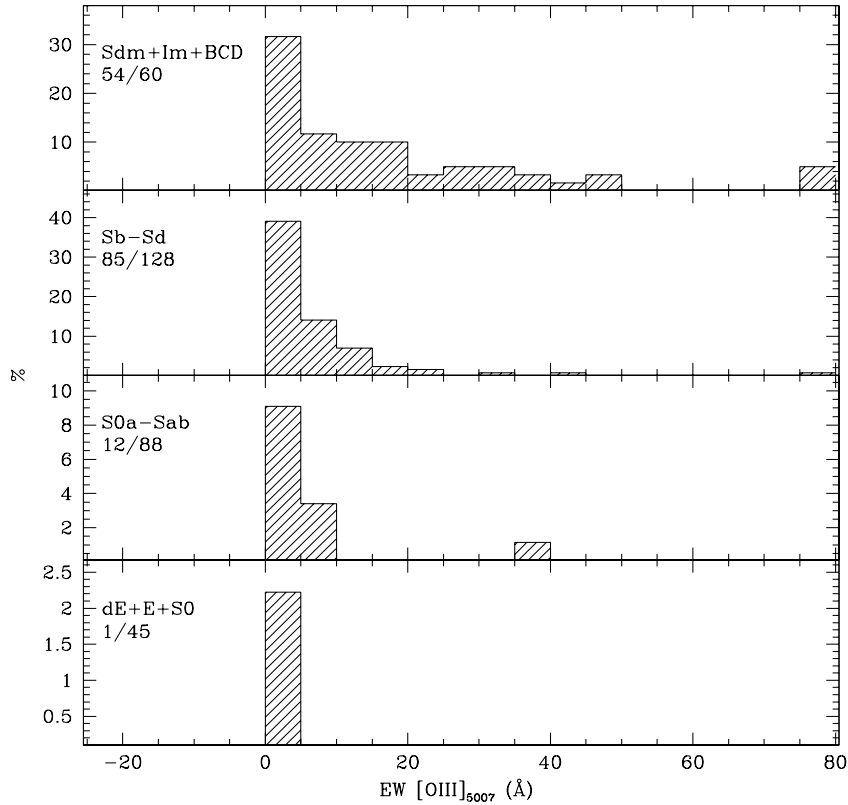

Fig. 13. Same as in Fig. 11 for [OIII2] $E W$.

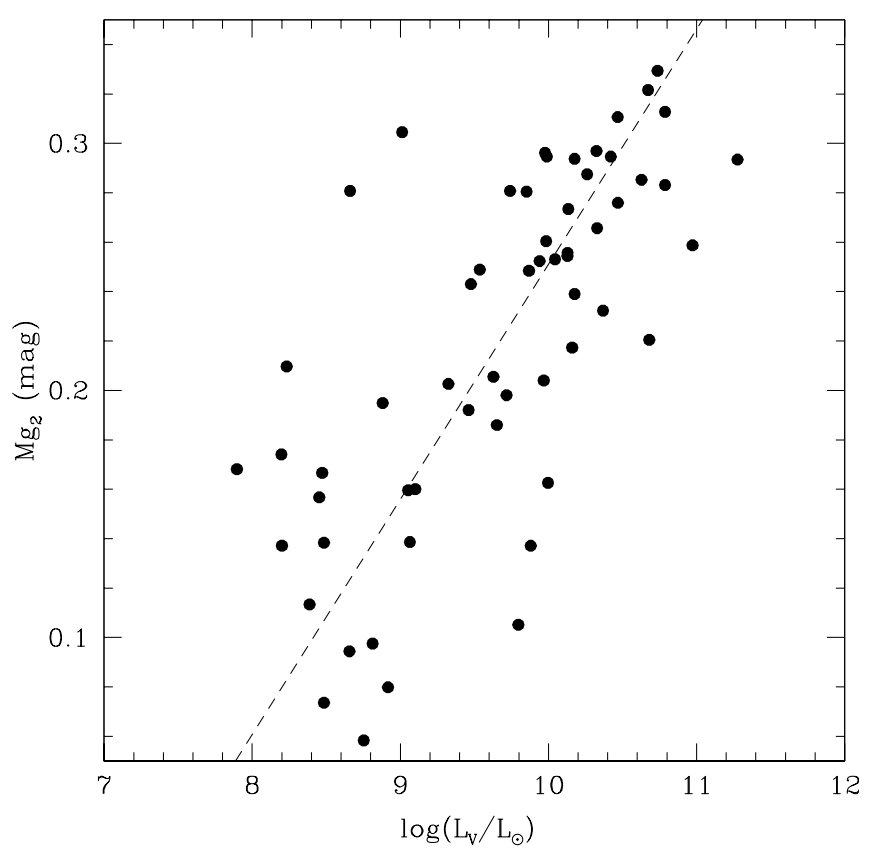

Fig. 14. The $\mathrm{Mg}_{2}$ absorption index vs. $V$ band luminosity for dE-dSO-E-SO. The dashed line represents the best linear fit to the data.

Column 3: $G_{4300}(\lambda \lambda$ 4283-4317) in $\AA$.

Column 4: $\mathrm{H} \beta(\lambda \lambda$ 4849-4877) in $\AA$.

Column 5: $\mathrm{Mg}_{2}(\lambda \lambda$ 5156-5197) in mag.

Column 6: NaD ( $\lambda \lambda$ 5879-5911) in $\AA$.

The principal metallicity index, $\mathrm{Mg}_{2}$ is plotted in Fig. 14 as a function of the $V$ band luminosity, confirming the well known increase of metallicity with luminosity (Bica \& Alloin 1987). Other absorption line indices, namely $\mathrm{NaD}, G_{4300}$ and $\Delta_{4000}$ are plotted in Figs. 15, 17 and 18 as a function of $\mathrm{Mg}_{2}$, showing the expected correlation with metallicity. On the opposite the strength of $\mathrm{H} \beta$ in absorption shows the reverse trend with $\mathrm{Mg}_{2}$ (Fig. 16), as this line is more sensitive to the age than to the 


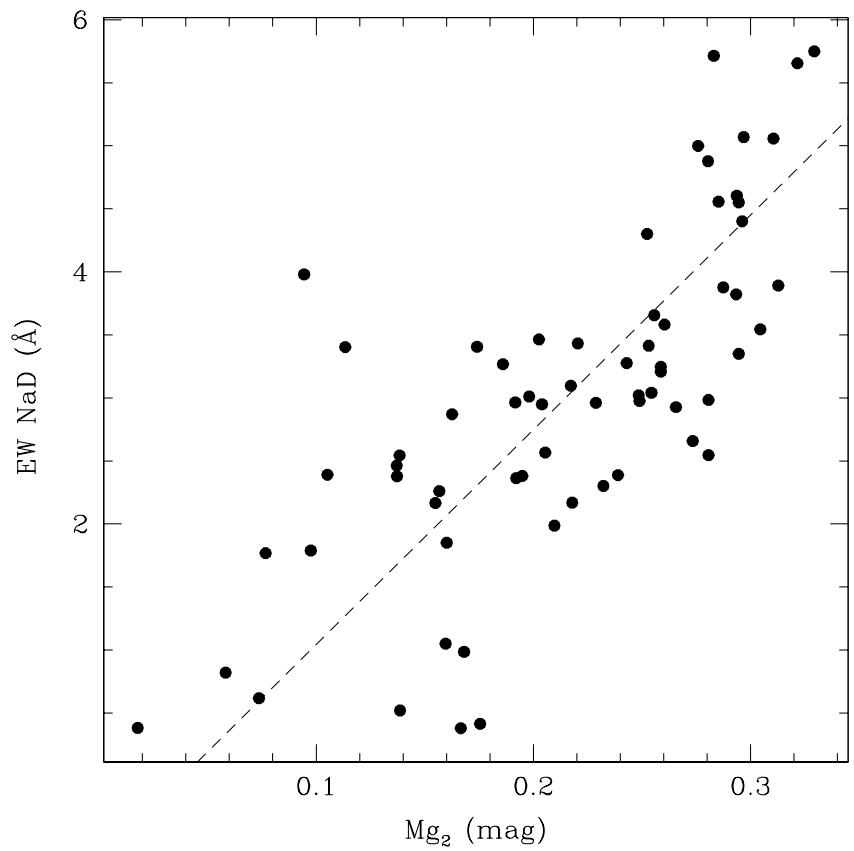

Fig. 15. The $\mathrm{Mg}_{2}$ absorption index vs. the $\mathrm{NaD}$ absorption index for dE-dSO-E-S0. The dashed line represents the best linear fit to the data.

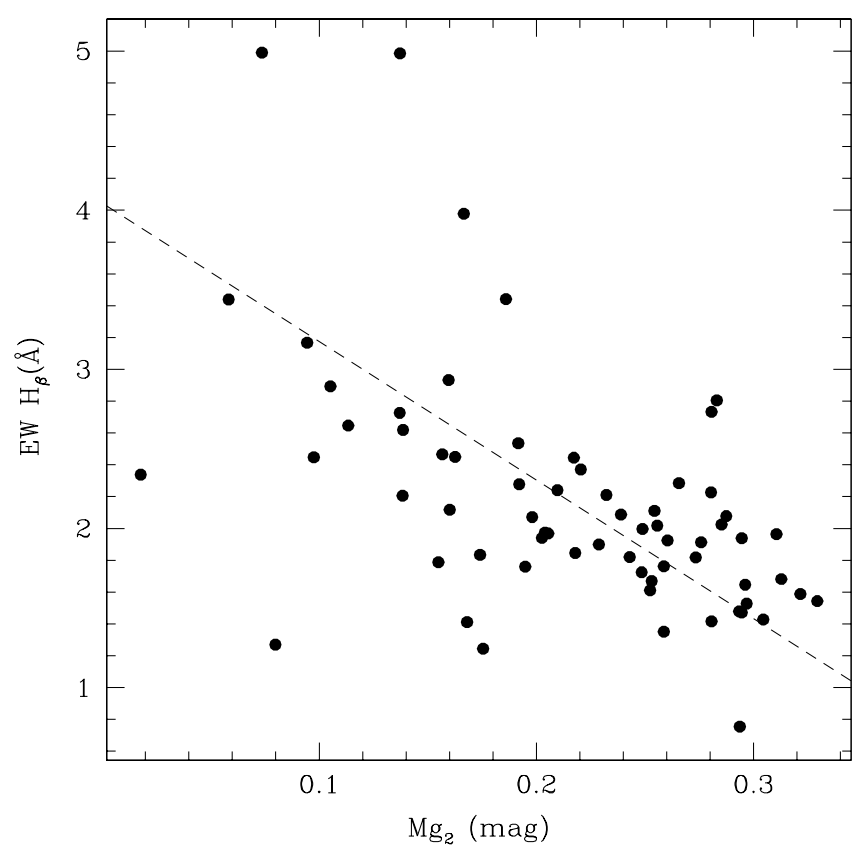

Fig. 16. The $\mathrm{Mg}_{2}$ absorption index vs. the $\mathrm{H}_{\beta}$ absorption index for dE-dS0-E-S0. The dashed line represents the best linear fit to the data.

metallicity of stellar populations (Worthey et al. 1994). The significance of these correlations can be estimated from the uncertainty in the slope and zero point listed in Table 4 . Beside the mentioned dependence on luminosity, no significant differences are found among $\mathrm{dE}$ and dS0 or among E and S0.

\section{Summary and conclusions}

Using 5 middle-size telescopes for 50 nights distributed over 6 years we obtained drift-scan spectra (3600-6800 A) with

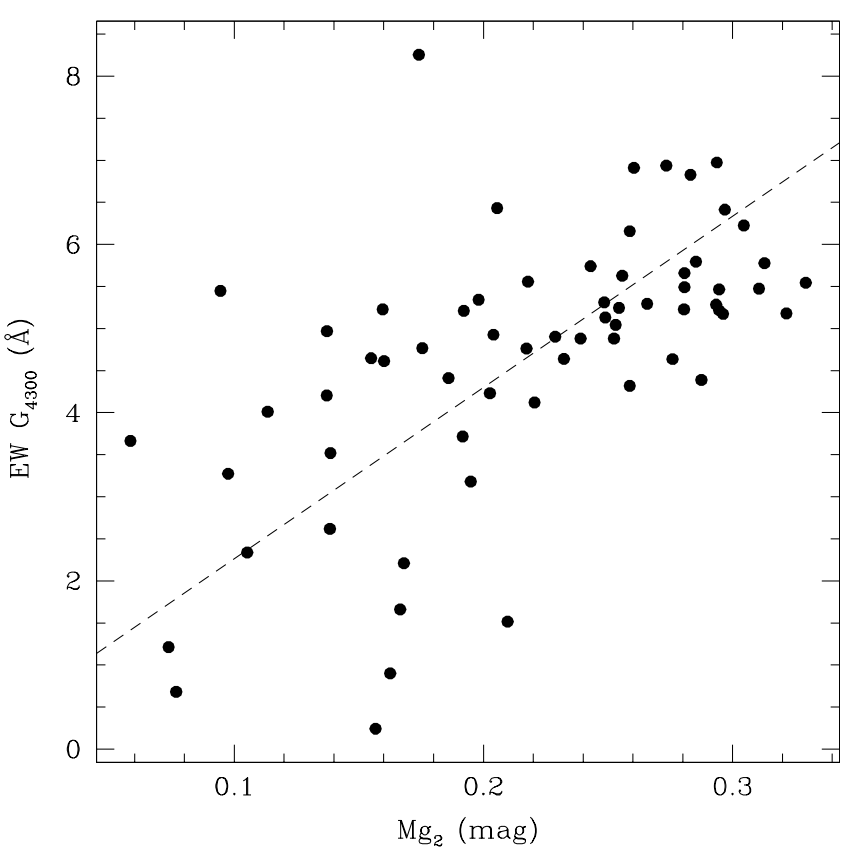

Fig. 17. The $\mathrm{Mg}_{2}$ absorption index vs. the $G_{4300}$ absorption index for dE-dS0-E-S0. The dashed line represents the best linear fit to the data.

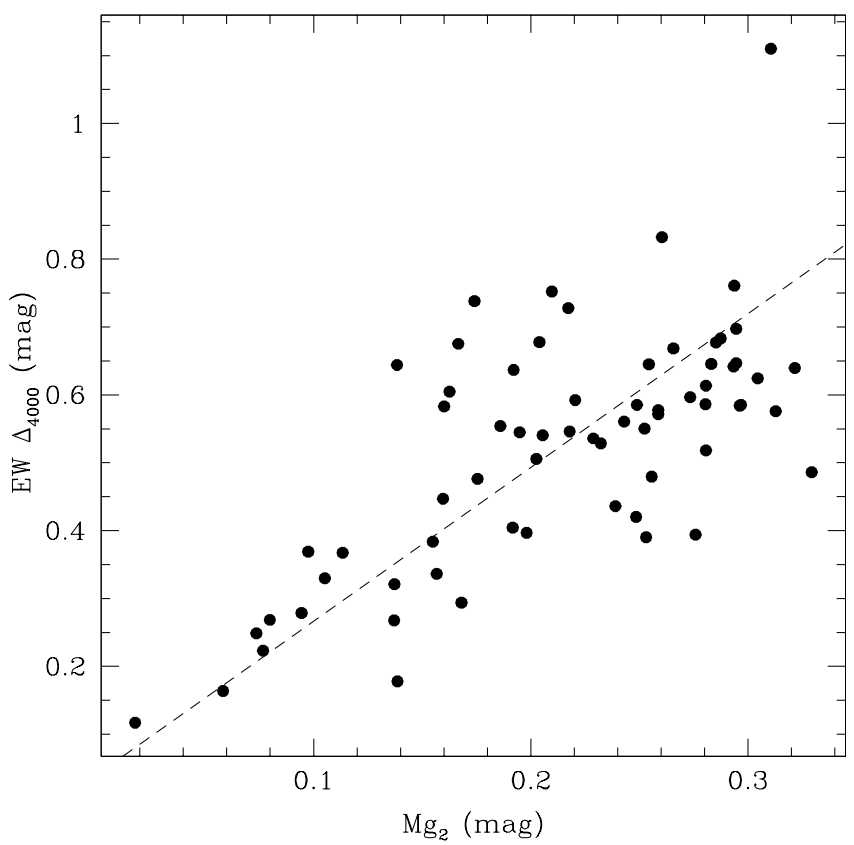

Fig. 18. The $\mathrm{Mg}_{2}$ absorption index vs. the Calcium break $\left(\Delta_{4000}\right)$ for $\mathrm{dE}-\mathrm{dS} 0-\mathrm{E}-\mathrm{S} 0$. The dashed line represents the best linear fit to the data.

$500<R<1000$ for 333 galaxies in nearby clusters. The majority (225) were secured for galaxies in the Virgo cluster. The observations can be considered representative of the spectral properties of giant and dwarf galaxies in this cluster, as the completeness achieved at $M_{p}=-15$ is $36 \%$ for all types and $51 \%$ for late-type galaxies.

Here we present the individual spectra reduced to their restframe wavelength and normalized to their intensity at $5500 \AA$. 
G. Gavazzi et al.: Spectro-photometry of galaxies in the Virgo cluster. II.
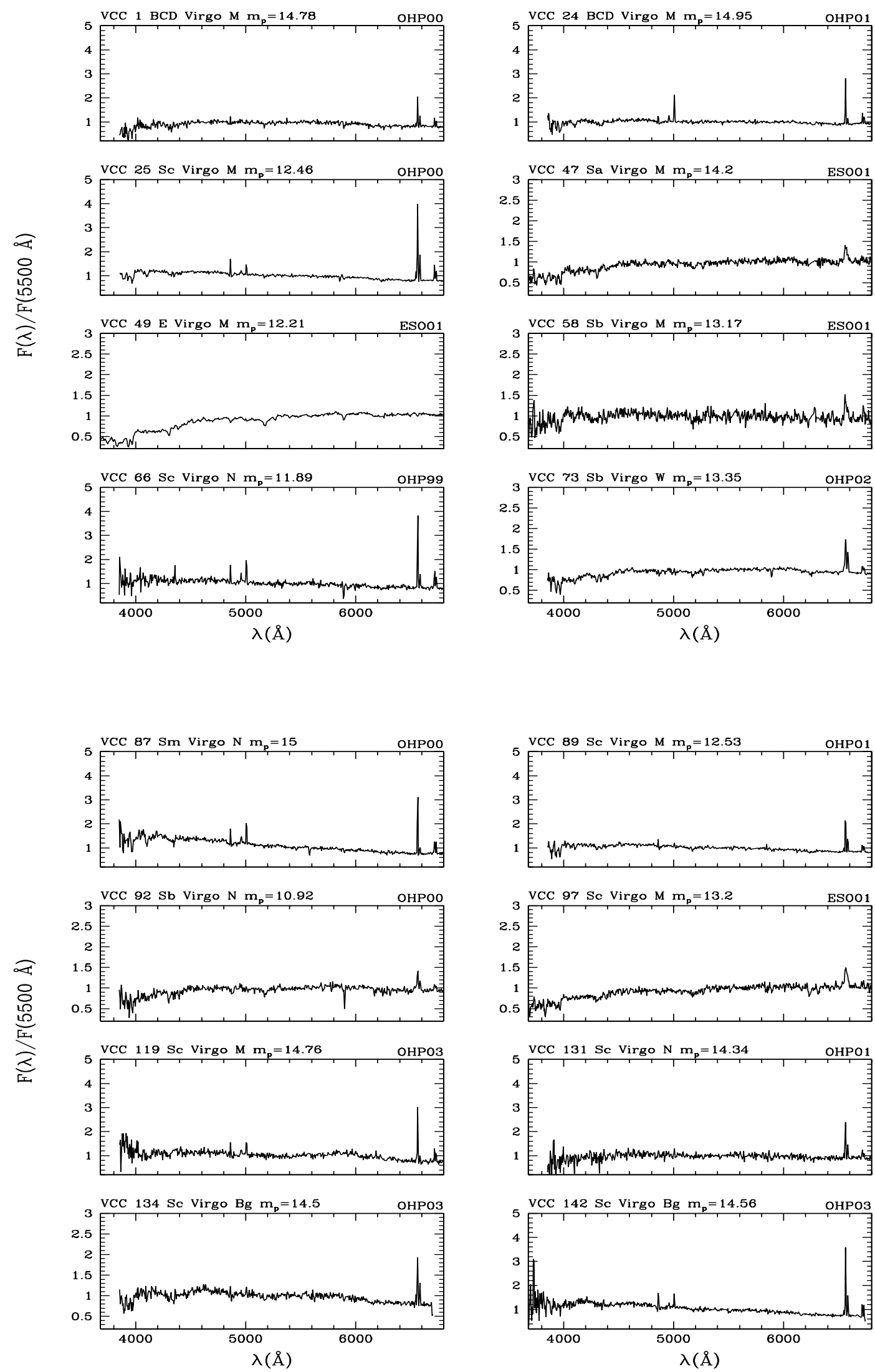

Fig. 19. The observed spectra. The galaxy identification, morphological type, membership, photographic magnitude and observing run are labeled on each panel (one page sample). 
Intensities (corrected for dereddening) and $E W \mathrm{~s}$ are derived for the principal lines both in emission and in absorption. Special care is devoted to deblending of $\mathrm{H}_{\alpha}$ from the [NII] doublet and of emission lines from underlying absorption. In the case of $\mathrm{H} \beta$ we measured underlying absorption $87 \%$ of the times we detected emission, with a mean $E W$ of $5.7 \AA$.

For early-type galaxies we derive the Lick absorption line indices.

The complete line analysis is postponed to a forthcoming Paper III where the metallicity indicators will be derived and analyzed.

The comparison of the line properties of our cluster sample with those of 200 isolated galaxies observed by Jansen et al. (2000) with a similar experimental setup will allow to study the influence of the cluster environment on the interstellar medium and on the stellar population of galaxies. This analysis will help shed light on the elusive processes that made cluster galaxies evolve separately from their isolated counterparts.

Acknowledgements. This research has made use of the NASA/IPAC Extragalactic Database (NED) and of the GOLDMine database. NED is operated by the Jet Propulsion Laboratory, California Institute of Technology, under contract with the National Aeronautics and Space Administration. GOLDMine is operated by the Universita' degli Studi di Milano-Bicocca.

\section{References}

Bica, E., \& Alloin, D. 1987, A\&A, 181, 270

Binggeli, B., Sandage, A., \& Tammann, G. 1985, AJ, 90, 1681

Binggeli, B., Popescu, C. C., \& Tammann, G. A. 1993, A\&AS, 98, 275

Boselli, A., \& Gavazzi, G. 2002, A\&A, 386, 124

Boselli, A., Gavazzi, G., Iglesias-Paramo, J., \& Vilchez, P. 2002, A\&A, 386, 134

Bruzual, G., \& Charlot, S. 1993, ApJ, 405, 538
Buat, V., Boselli, A., Gavazzi, G., \& Bonfanti, C. 2002, A\&A, 383, 801

Cardelli, J. A., Clayton, G. C., \& Mathis, J. S. 1989, ApJ, 345, 245

Feigelson, E. D., \& Babu, G. J. 1992, ApJ, 397, 55

Gavazzi, G., \& Boselli, A. 1996, Astro. Lett. Comm., 35, 1

Gavazzi, G., Pierini, D., \& Boselli, A. 1996, A\&A, 312, 397

Gavazzi, G., Boselli, A., Scodeggio, M., Pierini, D., \& Belsole, E. 1999, MNRAS, 304, 595

Gavazzi, G., Bonfanti, C., Sanvito, G., Boselli, A., \& Scodeggio, M. 2002a, ApJ, 576, 135 (Paper I)

Gavazzi, G., Boselli, A., Pedotti, P., Gallazzi, A., \& Carrasco, L. 2002b, A\&A, 386, 114

Gavazzi, G., Boselli, A., Donati, A., Franzetti, P., \& Scodeggio, M. 2003, A\&A, 400, 451

Jansen, R., Fabricant, D., Franx, M., \& Caldwell, N. 2000, ApJS, 126, 331

Jarrett, T. H., Chester, T., Cutri, R., Schneider, S. E., \& Huchra, J. P. 2003, AJ, 125, 525

Kennicutt, R. 1992, ApJ, 388, 310 (K92)

Kennicutt, R. 1998, ARA\&A, 36, 189

Kennicutt, R. C., Armus, L., Bendo, G., et al. 2003, PASP, 115, 928

Lequeux, J., Rayo, J. F., Serrano, A., Peimbert, M., \& Torres-Peimbert, S. 1979, A\&A, 80, 155

Osterbrock, D. 1989, Astrophysics of Gaseous Nebulae and Active Galactic Nuclei, University Science Books (Mill Valley)

Popescu, C. C., Tuffs, R. J., Volk, H. J., Pierini, D., \& Madore, B. F. 2002, ApJ, 567, 221

Raimann, D., Storchi-Bergmann, T., Bica, E., Melnick, J., \& Schmitt, H. 2000, MNRAS, 316, 559

Roberts, M., \& Haynes, M. 1994, ARA\&A, 32, 115

Seaton, M. J. 1979, MNRAS, 187, 73

Savage, B., \& Mathis, J. 1979, ARA\&A, 17, 73

Stasinska, G., \& Sodre, L. Jr. 2001, A\&A, 374, 919

Stoughton, C., Lupton, R. H., Bernardi, M., et al. 2002, AJ, 123, 485

Tuffs, R. J., Popescu, C. C., Pierini, D., et al. 2002, ApJS, 139, 37

Worthey, G., Faber, S. M., Gonzales, J. J., \& Burstein, D. 1994, ApJS, 94, 687

Zwicky, F., Herzog, E., Karpowicz, M., Kowal, C., \& Wild, P. 1961-1968, Catalogue of Galaxies and of Cluster of Galaxies (Pasadena: California Institute of Technology, CGCG) 